\title{
A novel quantum-inspired evolutionary view selection algorithm
}

\author{
SANTOSH KUMAR $^{1}$ and T V VIJAY KUMAR ${ }^{2, *}$ \\ ${ }^{1}$ Department of Computer Science and Engineering, ABES Engineering College, Ghaziabad, India \\ ${ }^{2}$ School of Computer and Systems Sciences, Jawaharlal Nehru University, New Delhi, India \\ e-mail: santoshg25@gmail.com; tvvijaykumar@hotmail.com
}

MS received 6 April 2016; accepted 20 June 2018; published online 29 August 2018

\begin{abstract}
A data warehouse ( $D W$ ) is designed primarily to meet the informational needs of an organization's decision support system. Most queries posed on such systems are analytical in nature. These queries are long and complex, and are posed in an exploratory and ad-hoc manner. The response time of these queries is high when processed directly against a continuously growing $D W$. In order to reduce this time, materialized views are used as an alternative. It is infeasible to materialize all views due to storage space constraints. Further, optimal view selection is an NP-Complete problem. Alternately, a subset of views, from amongst all possible views, needs to be selected that improves the response time for analytical queries. In this paper, a quantum-inspired evolutionary view selection algorithm (QIEVSA) that selects Top- $K$ views from a multidimensional lattice has been proposed. Experimental comparison of QIEVSA with other evolutionary view selection algorithms shows that QIEVSA is able to select $T o p-K$ views that are comparatively better in reducing the response times for analytical queries. This in turn aids in efficient decision making.
\end{abstract}

Keywords. Data warehouse; on-line analytical processing; materialized view selection; quantum-inspired evolutionary algorithm.

\section{Introduction}

Ever since the invention of computers, and especially since their ever-increasing acceptance with businesses, almost all organizations have nowadays computerized their business operations. Business data are collected and stored using computers. Managing increasing volumes of digital data was a tricky proposition from the very beginning. On-Line Transaction Processing (OLTP) systems were developed to collect transaction data [1]. These systems were designed with complex structures in order to minimize data redundancies on account of very fast write operations. The informational value of this stored transactional data was recognized from the very beginning considering that many applications, based on them, for report generation, trend analysis, etc., were developed for decision support. OLTPs were not designed for fast retrieval operations of huge volumes of data for their analysis; hence it performed very limited analysis and that to inefficiently $[1,2]$. With the advent of computer networks having multiple communication protocols, computers with varied hardware configurations, distinct operating systems and disparate database management systems, the stand-alone transactional data sources of an organization became incompatible and obsolete. As a result, the credibility of an organization's

*For correspondence data asset for analysis diminished and data analysis using incompatible data sources became an exorbitantly costly process, causing information bottlenecks in the organization [3]. A data warehouse $(D W)$ was designed as an alternative to address this crisis with the aim of meeting the informational needs of the organization's decision support system $(D S S)$ [4]. $D W$ is a centralized repository of historic, integrated, subjected-oriented, time-variant and non-volatile data, created and maintained for supporting complex data analysis, for acquiring information to support strategic and tactical decision making [1-3]. In the $D W$, data from various disparate, remote, heterogeneous and incompatible transactional data sources are extracted, transformed, consolidated and integrated to obtain correct, unambiguous, consistent and complete data. In transactional data sources, data are organized and stored around specific operational applications; however, in a $D W$, data are organized and stored not by their applications, but by business subjects crucial for the organization $[2,3,5]$. Generally, operational systems store the current value of data by overwriting its previous value; they do not maintain a record of all its preceding values. As a result, historical analysis cannot be performed using such operational data. In contrast, a $D W$, in addition to the current value of data, stores all values ever assigned to the data since its creation. The data that enter a $D W$ are never deleted, but stored permanently throughout the life of the organization. Such time-variant 
and non-volatile data facilitates historical and predictive analysis. A $D W$ stores atomic data, as well as summarized data, to support detailed and fast analytical queries.

The sequence of interactive and multi-perspective data analysis performed on the $D W$ using some data analytical tool is called On-Line Analytical Processing (OLAP), as this is the primary goal of building a $D W$ [2, 6-8]. OLAP queries access very large volumes of historical and aggregate data at different levels of granularity using roll-up and drill down OLAP operators along certain combinations of dimensions (sets of attributes). They use slice and dice $O L A P$ operators to select and project a desired portion of data. A pivot operator is used by them to reorient the data to another view. These complex OLAP operations are made possible by the multidimensional data model [6-8]. It provides a multidimensional view of the data comprising measures and dimensions [9]. A measure is the numerical quantification of an event of interest and a dimension is a set of attributes forming the context of a measure [9]. Product, Customer and Time can be dimensions for a sales measure as shown in figure 1. The multidimensional data model is implemented using star schema in relational database management systems. It is implemented using multidimensional arrays in a multidimensional database [9]. The star schema with Sales as a fact table and Customer, Product and Time as the dimensional tables is shown in figure 2.

One of the major challenges during an OLAP session is the speed with which the $D W$ and $O L A P$ components of a $D S S$ are able to respond to $O L A P$ queries posed by analysts. Although the available $O L A P$ operators have been successfully assisting such queries, if DSS takes hours or days to answer these, the analysis would be very limited and less productive as response delays would break the chain of adhoc analytical thoughts of an analyst and the information obtained at the end of such a long OLAP sessions would mostly be obsolete and irrelevant. Not only the depth and width of the information are essential but also the speed at which such information is delivered is all the more important to stay competitive in a dynamic market environment having many other competitors [3]. OLAP queries

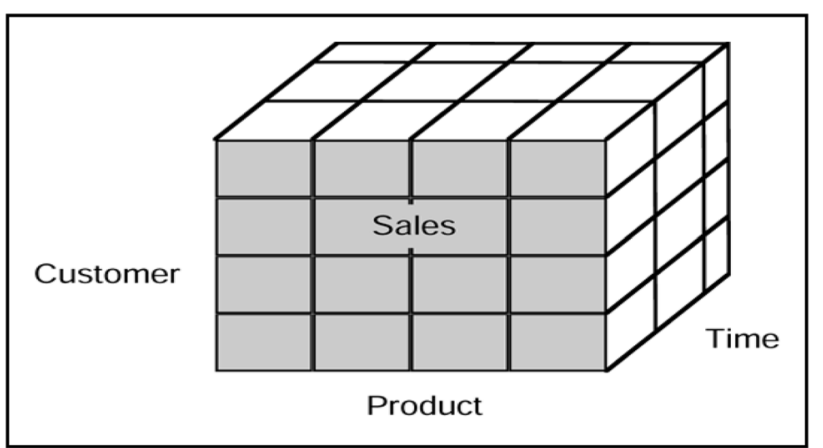

Figure 1. Data cube.

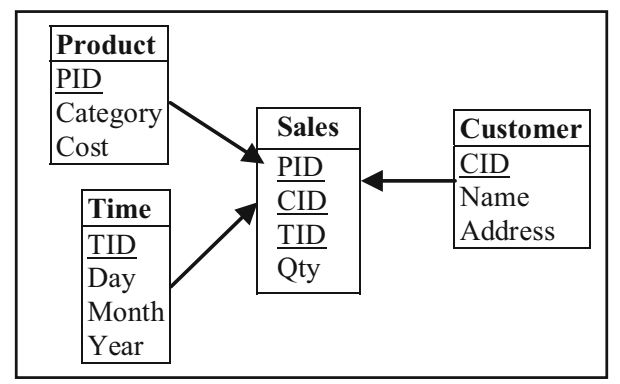

Figure 2. Star schema.

are usually long, complex, exploratory and ad hoc in nature. These queries when posed against a voluminous $D W$ consume lot of time for their processing, thereby increasing the query response time. This problem worsens due to the continuously growing data in a $D W$, as these queries may take hours and days to process, where the desired requirement is of only a few seconds and minutes. Although several query optimization techniques and indexing strategies [10-14] exist, they do not scale up with the ever increasing voluminous data in the $D W$. Scalability can be addressed by pre-computing relevant and frequently accessed data in the $D W$ and storing it separately as materialized views [15] in the $D W$. This pre-computed information, which is significantly small when compared with the voluminous $D W$, can be used to process $O L A P$ queries in an efficient manner.

Materialized views, unlike virtual views, store data along with their query definition. Their primary purpose is to reduce the response time of OLAP queries. Amongst the issues associated with materialized views like view maintenance, view synchronization, view adaptation, answering queries using views and view selection [12], view selection is the focus of this paper and is discussed next.

\subsection{View selection}

View selection is concerned with the selection of an appropriate subset of views from amongst all possible views for a given query workload that can improve the query performance, while conforming to the resource constraints in terms of storage space, maintenance cost, etc. [16-20]. It has been studied in the context of query optimization, warehouse design, distributed databases, semantic web databases, etc. [17, 21-23]. According to [20], view selection is concerned with the selection of views, for a given query workload, from a database that is capable of being stored within the available storage space. Since the number of views is exponential with respect to the number of dimensions, all possible views cannot be materialized due to storage space constraint. Thus, there is a need to select a subset of views that conform to the storage space constraints from amongst all possible views. Further, 
optimal selection of subse $\mathrm{t}$ of views is a NP-Complete problem [13]. The objective then is to select a subset of good quality views, if not optimal, for materialization. This subset cannot be arbitrarily selected, as it may result in selecting views that are irrelevant and not capable of answering the OLAP queries. Several approaches exist that have been attempted to select this subset of views, which are broadly classified as empirically based or heuristically based [24]. The empirically based view selection approach [24-33] considers data accessed by past queries as indicators of data that are likely to be accessed by future queries. These approaches monitor and assess these queries on parameters like the query frequency, data accessed, etc., and use this information to select subsets of views from materialization. On the other hand, heuristically based view selection approaches use heuristics, to prune the search space of all possible views to select views that can reduce the response time of $O L A P$ queries. Most of these are based on greedy heuristics [12, 13, 34-50]. Among these, most of them focus on the issues and problems associated with the greedy view selection algorithm given in [13], also referred to as HRUA [36, 37, 43-49]. This paper also proposes a view selection algorithm that attempts to address the issues and problems associated with the greedy view selection algorithm $H R U A$ given in [13].

$H R U A$, in each iteration, computes the benefit of each view of a multidimensional lattice, using its size, and selects amongst them the one having maximum benefit. For selecting Top- $K$ views, this process continues for $K$ number of iterations. HRUA aims to select the Top- $K$ views that minimize the total cost of evaluating all the views. However, HRUA has certain limitations. Since the number of views is exponential with respect to the number of dimensions, increase in the number of dimensions leads to a deterioration in the quality of views selected using $H R U A$ with respect to TVEC. This is because the possible number of views from which to select $T o p-K$ views increases with increase in the number of dimensions. Thus $H R U A$, being a greedy algorithm, selects a sub-optimal quality of views. Another limitation with $H R U A$ is that it is not scalable for higher dimensions, i.e., it becomes computationally infeasible to select views for higher dimensional data sets. These limitations need to be addressed in order to select good quality views for higher dimensional data sets. One way to address this problem is by selecting views using evolutionary algorithms. Darwin's theory of evolution by means of natural selection has been the heuristic used in evolutionary algorithms [51]. According to it, genetically and behaviourally well adapted progenies survive and replace their parents to reproduce in order to prolong the existence of their species. Many aspects of natural evolution have been viewed as computational processes and have been adapted with stochastic elements to solve complex computational problems [51]. These algorithms have been used for view selection. Evolutionary view selection is discussed next.

\subsection{Evolutionary view selection}

Evolutionary view selection would select views by exploring and exploiting the search space of all possible views using evolutionary operators like selection, crossover and mutation with an aim to select good sets of views for materialization. Several evolution-based view selection algorithms exist [52-63]. Reference [62] used a genetic algorithm $(G A)$ to select an optimal set of views, for a given set of multiple query processing plans, to minimize its processing cost. The proposed $G A$ performed better than heuristics of [62] and [18]; it was also observed that the hybrid of $G A$ and heuristics of [62] and [18] performed better than the $G A$ and heuristics of [62] and [18]. Reference [52] used the Genetic Local Search $(G L S)$ algorithm using AND-OR view graph to address the view selection problem. The GLS searches in two steps; in the first step it uses a local search to improve the population of chromosomes, whereupon the $G A$ is employed to diversify the search to look out for an unexplored solution space. It was applied to a real database to determine its efficiency. $G A$, in conjunction with OR view graph, was used for the maintenance-cost view selection problem [54]. The results obtained were consistently within 10 percent of the optimal solution; further, it exhibited a linear execution time. Reference [61] proposed a two-level structure for materialized view selection and used evolutionary algorithms with Multiple View Processing Plan (MVPP) framework to select views. It was observed that evolutionary algorithms had impractically long computation time and the quality of solutions was no better than those of the hybrid of evolutionary algorithms and heuristics of [61] and [18]. Reference [60] proposed a stochastic ranking evolutionary algorithm (SEA) for the maintenance cost view selection problem. In finding optimal solutions, it was observed that $S E A$ was able to obtain near-optimal results that were very close to those obtained by $\mathrm{A}^{*}$-heuristic; it was also noticed that $S E A$ performed much better than the algorithm proposed by [54]. A* and $S E A$ performed better than the algorithm proposed by [54] and the inverted-tree greedy for smaller instances of the problem. As $\mathrm{A}^{*}$ and inverted-tree greedy were not able to handle lattices with 256 nodes, SEA and the algorithm of [54] were compared for higher dimension data sets; it was observed that the solutions obtained by $S E A$ were much better than those obtained through the algorithm of [54], though it took longer time than the algorithm of [54]. Reference [55] proposed a genetic greedy method to select a set of materialized cubes from the data cube; it included a greedy repair procedure to handle infeasible solutions generated by simple GA. Its performance was compared to that of the greedy algorithm of [13] and was observed to be better than the latter. Niched Pareto Genetic Algorithm (NPGA) and Multi-Objective Genetic Algorithm (MOGA) have been applied to the maintenance cost view selection problem in [53] and the solutions are compared to those produced by HRUA. Their 
performance was studied using real and synthetic data sets. It was observed that the performances of NPGA and MOGA were better than that of $H R U A$ for all problems, but their performance gap decreased for larger instances of the problem. The performances of NPGA and MOGA were almost similar for most of the problems, but MOGA performed better than NPGA for skewed problems. Reference [56] proposed $M-O L A P$ Genetic and $M-O L A P$ co-Genetic algorithm to select distributed $O L A P$ cubes for materialization. They were shown to perform better than the greedy algorithm. Reference [57] applied GA to the view selection problem with dual constraints, i.e., space and maintenance cost constraints. The benefit of the solutions obtained by $G A$ was at least 64 percent of the benefit obtained of the optimal solution. The results of $G A$ were compared with those of exhaustive search and it was observed that the results of $G A$ were better than the latter. Reference [58] used the evolutionary algorithm to address the weighted materialized view selection problem, which included both the volume and importance of the retrieved data. Simulation results showed that it performed substantially better than the brute force method and the heuristic approach in terms of the quality of the solutions and execution time. In [64], a GA-based view selection algorithm GVSA was proposed, which was used to select Top- $K$ views from a multidimensional lattice. GVSA was shown to perform better than HRUA. Further, in [65], memetic-based view selection algorithm MVSA was proposed, which incorporated iterative improvement into the evolutionary nature of the algorithm. MVSA produced better quality Top-K views when compared with those produced using GVSA [66]. Later, in [66], algorithm DEVSA that selects Top-K views from a multidimensional lattice using differential evolution was proposed. DEVSA, in comparison with GVSA and $M V S A$, was able to select comparatively better quality views.

The effectiveness of evolutionary algorithms depends upon their ability to achieve an appropriate balance between exploration and exploitation of the search space, while arriving at solutions to a given problem. Though the traditional evolutionary algorithms are characterized by individual representation, fitness function, selection, crossover and mutation, they are not able to achieve better population diversity and thus are unable to explore and exploit the search space adequately [67]. A quantum-inspired evolutionary algorithm (QIEA) has been used as an alternative to achieve such population diversity using the principles of quantum computing such as quantum bit and superposition of states $[67,68]$. In $Q I E A$, an individual is probabilistically represented using a string of $Q$-bits, where each $Q$-bit is the smallest unit of information. Since the $Q$ bit individual can represent binary solutions probabilistically, it can ensure better population diversity, which in turn would enable better exploration and exploitation of the search space [67]. Further, the $Q$-gate operation in $Q I E A$ maintains an appropriate balance between the exploration and the exploitation of the search space and thereby drives the individuals towards better solutions [68, 69]. QIEA has been shown to perform better than the traditional evolutionary algorithms [67, 70-74]. In this paper, an attempt has been made to use QIEA to address the view selection problem in the context of a multidimensional lattice framework. Accordingly, a quantum-inspired-evolutionbased view selection algorithm (QIEVSA) that selects the $T o p-K$ views from a multidimensional lattice has been proposed. Further, QIEVSA is compared to the existing view selection algorithms GVSA, MVSA and DEVSA. Such comparisons show that QIEVSA performs comparatively better than GVSA, MVSA and DEVSA.

\subsection{Organization of the paper}

This paper is organized as follows: view selection using QIEA is discussed in section 2 followed by an illustrative example in section 3. Experimental results are given in section 4. Section 5 presents the conclusion.

\section{View selection using $Q I E A$}

Since the proposed view selection algorithm focuses on $H R U A$, it considers a multidimensional lattice for view selection. In a multidimensional lattice framework, the root node represents the fact table while the intermediate nodes represent all other possible combinations of the dimension tables (views). Multidimensional lattice is discussed next.

\subsection{Multidimensional lattice}

An OLAP cube, shown in figure 1, can be represented as a multidimensional lattice shown in figure 3. This lattice of figure 3 comprises three dimensions, viz., Customer $(C)$, Product $(P)$ and Time $(T)$, and therefore the total number of possible views is $8\left(2^{3}\right)$. The possible views are represented by nodes of the lattice with the view index in parenthesis and the view size alongside. The dependences between the

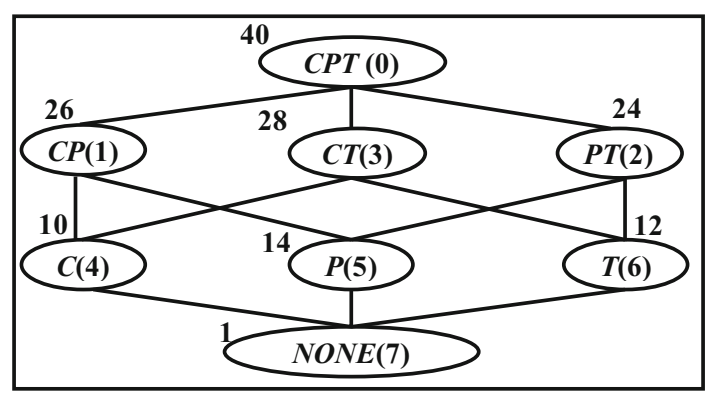

Figure 3. Three-dimensional lattice of views along with the size of each view. 
views are represented using edges and each view is either directly or indirectly dependent on the root view, which represents the base fact table CPT. View NONE has no dependent view. View $C P$ is directly dependent on view $C P T$, whereas view $C$ is indirectly dependent on view $C P T$.

Next, the QIEA that has been discretized and adapted for view selection is discussed.

\subsection{QIEA}

Quantum computation is based on the physical principles of quantum mechanics, like $Q$-bits, superposition, $Q$-gates and quantum measurement, for solving problems in the context of classical computing paradigms [68]. In quantum computer systems, a single bit of information, i.e., a quantum bit (Q-bit), may be in the "0" state or in the "1" state or in any superposition of the " 0 " state and " 1 " state [67]. Its state can be given as [67]

$$
|\Psi>=\alpha| 0>+\beta \mid 1>
$$

where $10>$ and $11>$ represent the values of classical bits 0 and 1 , respectively, and $\alpha$ and $\beta$ are complex numbers satisfying:

$$
|\alpha|^{2}+|\beta|^{2}=1
$$

$|\alpha|^{2}$ and $|\beta|^{2}$ are the probability of the $Q$-bit being in "0" state and "1" state, respectively.

The state of the Q-bit can be changed using a quantum gate (Q-gate) that is a reversible gate represented by a unary operator " $U$ "; when it acts on the Q-bit it satisfies the condition $\mathrm{U}^{+} \mathrm{U}=\mathrm{UU}^{+}$[67]. Several Q-gates exist like NOT gate, controlled NOT gate, rotation gate, etc. $[67,75]$. For a system with $m$ Q-bits, there are $2^{m}$ states. Observing a quantum state collapses it to a single state [67].

Quantum computers were formalized in the late 1980s and active research was carried out in the 1990s, during which research related to merging of quantum computing and evolutionary computing started. Accordingly, several algorithms exist that are broadly classified [68] as evolution-designed quantum algorithms [76-80], quantum evolutionary algorithms [81-86] and QIEAs [67, 87-93]. This paper focuses on the use of the QIEA to address the view selection problem.

QIEA uses probabilities associated with each state to describe the behaviour of the system. QIEA uses Q-bits, Q-gates and the observation process. Q-bits represent individuals, Q-gates operate on Q-bits to generate offsprings and the observation process enables the quantum particle to take state $\mid 0>$ or state $\mid 1>$. The superposition state represented by Q-bits would collapse to a single state during the observation process. QIEA uses Q-bit representation, which is probabilistically a linear superposition of states, to describe individuals of the population. It uses $Q$-gate to generate individuals with better solutions for the next generation. It can also exploit the search space for a global solution having, even, a single element. Several QIEA algorithms exist and are broadly classified into three types, namely binary observation QIEA (bQIEA) [67, 89, 90], real observation QIEA (rQIEA) [54, 93] and QIEA-like algorithms (iQIEA) [87, 88, 92]. In this paper, bQIEA [67] has been used to address the view selection problem.

An individual in bQIEA is represented as a string of Qbits as follows:

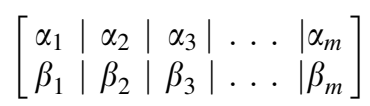

where $\left|\alpha_{i}\right|^{2}+\left|\beta_{i}\right|^{2}=1, i=1,2, \ldots, m$, where $m$ is the number of Q-bits representing an individual.

The algorithm $b Q I E A$, as proposed in $[67,68]$, is given in figure 4.

In Step-1, the generation number $g$ is initialized to $1 ; \alpha$ and $\beta$ values in quantum state $Q(g)$ are initialized in a manner such that $|\alpha|^{2}+|\beta|^{2}=1$. As a result, each Q-bit individual is a linear superposition of all possible states. Next, in Step-2, a binary solution $P(g)$ is obtained by observing the quantum state $Q(g)$. The resultant binary string of length $m$ is formed by selecting either 0 or 1 for each bit based on the probability $|\alpha|^{2}$ or $|\beta|^{2}$. In Step-3, each binary solution in $P(g)$ is evaluated based on the problem-specific fitness function. The initial best solutions in $P(g)$ are selected and stored in $B(g)$ and the best solution of $B(g)$ in $b$ in Step-4. In Step-5, $g$ is incremented by 1 . In Step-6, binary solutions in $P(g)$ are obtained by observing $Q(g-1)$, as in Step-2. The solutions in $P(g)$ are evaluated using the problem-specific fitness function in Step-7, as in Step-3. Thereafter, in Step 8, Q-bit individuals in $Q(g)$ are updated by applying rotation Q-gate as follows [67]:

$$
G^{g-1}(\theta)=\left[\begin{array}{cc}
\cos \theta^{g-1} & -\sin \theta^{g-1} \\
\sin \theta^{g-1} & \cos \theta^{g-1}
\end{array}\right]
$$

where $\theta^{g}$ is an adjustable Q-gate rotation angle.

The Q-bit $\left[\alpha^{g}, \beta^{g}\right]$ is updated as follows [67]:

$$
\left[\begin{array}{l}
\alpha^{g} \\
\beta^{g}
\end{array}\right]=G^{g-1}(\theta)\left[\begin{array}{l}
\alpha^{g-1} \\
\beta^{g-1}
\end{array}\right]
$$

where $\theta^{g-1}=s\left(\alpha^{g-1}, \beta^{g-1}\right) \Delta \theta^{g-1} ; s\left(\alpha^{g-1}, \beta^{g-1}\right) \quad$ and $\Delta \theta^{g-1}$ are the sign and the magnitude of the rotation gate, respectively. The afore-mentioned particular values are taken from the look-up table [68] given in figure 5; $x$ and $y$ are bits of $p(g)$ and $b$, respectively.

In Step-9, the best solutions, from among $B(g-1)$ and $P(g)$, are selected and stored in $B(g)$ and $b$ is updated with the best solution from amongst solutions in $B(g)$ and $b$. In Step-10, the migration condition is checked. If it is found to 


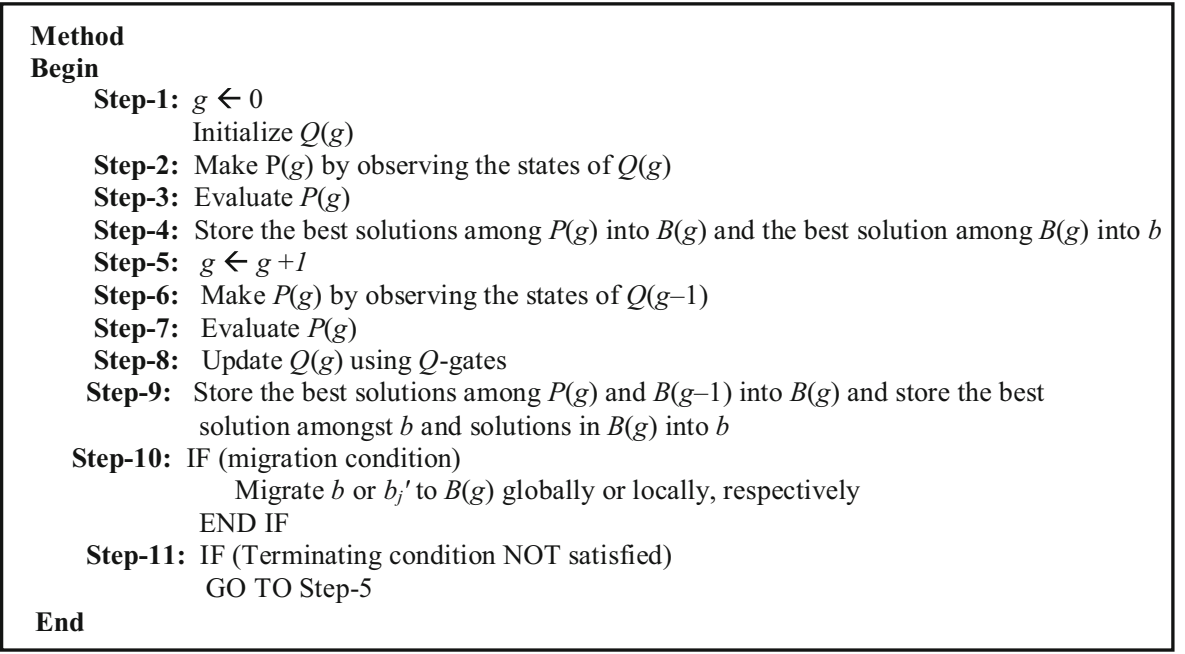

Figure 4. Algorithm $b Q I E A[67,68]$.

\begin{tabular}{|c|c|c|c|c|c|}
\hline \multirow{2}{*}{$\boldsymbol{x}$} & \multirow{3}{*}{$\boldsymbol{y}$} & \multirow{2}{*}{$\boldsymbol{f}(\boldsymbol{x}) \leq f(\boldsymbol{y})$} & \multirow{2}{*}{$\Delta \theta^{g-1}$} & \multicolumn{2}{|c|}{$s\left(\alpha^{g-1}, \beta^{g-1}\right)$} \\
\cline { 5 - 6 } & & & $\alpha^{g-1} \beta^{g-1} \geq 0$ & $\alpha^{g-1} \beta^{g-1}<0$ \\
\hline 0 & 0 & false & 0 & \pm 1 & \pm 1 \\
\hline 0 & 0 & true & 0 & \pm 1 & \pm 1 \\
\hline 0 & 1 & false & $0.01 \pi$ & 1 & -1 \\
\hline 0 & 1 & true & 0 & \pm 1 & \pm 1 \\
\hline 1 & 0 & false & $0.01 \pi$ & -1 & 1 \\
\hline 1 & 0 & true & 0 & \pm 1 & \pm 1 \\
\hline 1 & 1 & false & 0 & \pm 1 & \pm 1 \\
\hline 1 & 1 & true & 0 & \pm 1 & \pm 1 \\
\hline
\end{tabular}

Figure 5. Look-up table [68].

be satisfied, the best solution $b$ is migrated to $B(g)$ or some of the best solutions in $B(g)$ are migrated to other solutions in $B(g)$. Next, the termination condition is checked in Step11. If the termination condition is not satisfied, then Step-5 to Step-11 are repeated until the termination condition is satisfied.

The afore-mentioned algorithm $b Q I E A$ has been adapted to solve the view selection problem. Accordingly, a QIEVSA has been proposed that selects the Top-K views from a multidimensional lattice using bQIEA. QIEVSA is discussed next.

\subsection{QIEVSA}

QIEVSA [94] selects Top- $K$ views from a multidimensional lattice using $b Q I E A$. QIEVSA takes the $d$-dimensional lattice of views, number of views to be selected $K$ and the pre-specified number of generations $G$ for which QIEVSA runs as input, and produces the Top-K views $T K V$ as the output. QIEVSA comprises the following steps.

\section{Step-1: Initialization}

Initialize the generation $g=0$. Generate a random population $Q_{T K V}(g)$ of $N$ Top- $K$ views with quantum chromosome length $K d$, where each quantum gene is represented by $d$ quantum bits (Q-bits) and $d$ is the dimension of the lattice.

$$
Q_{T K V}(g)=\left\{q t k v_{1}^{g}, q t k v_{2}^{g}, \ldots, q t k v_{N}^{g}\right\}
$$

where

$$
q t k v_{i}^{g}=\left(\begin{array}{cccc|cccc|cc|cccc}
\alpha v_{i 11}^{g} & \alpha v_{i 12}^{g} & \ldots & \alpha v_{i 1 d}^{g} & \alpha v_{i 21}^{g} & \alpha v_{i 22}^{g} & \ldots & \alpha v_{i 2 d}^{g} & \ldots & \ldots & \alpha v_{i K 1}^{g} & \alpha v_{i K 2}^{g} & \ldots & \alpha v_{i K d}^{g} \\
\beta v_{i 11}^{g} & \beta v_{i 12}^{g} & \ldots & \beta v_{i 1 d}^{g} & \beta v_{i 21}^{g} & \beta v_{i 22}^{g} & \ldots & \beta v_{i 2 d}^{g} & \ldots & \ldots & \beta v_{i K 1}^{g} & \beta v_{i K 2}^{g} & \ldots & \beta v_{i K d}^{g}
\end{array}\right)
$$




$$
\begin{aligned}
& \left|\alpha v_{i j l}^{g}\right|^{2}+\left|\beta v_{i j l}^{g}\right|^{2}=1 \\
& \quad \forall i=1,2, \ldots, N ; j=1,2, \ldots, K ; l=1,2, \ldots, d .
\end{aligned}
$$

Initially, at $g=0$, all possible states are superimposed with the same probability, i.e.

$$
\begin{aligned}
\alpha v_{i j l}^{0} & =\frac{1}{\sqrt{2}} \text { and } \beta v_{i j l}^{0}=\frac{1}{\sqrt{2}} \\
\forall i & =1,2, \ldots, N ; j=1,2, \ldots, K ; l=1,2, \ldots, d .
\end{aligned}
$$

In this step of initialization of $Q_{T K V}(g)$, each of the Q-bits $\left(\alpha v_{i j l}^{g}\right.$ and $\left.\beta v_{i j l}^{g}\right)$ is initialized to $(1 / \sqrt{ } 2,1 / \sqrt{ } 2)$. Each Q-bit Top- $K$ views $q t k v_{i}^{g}$ represents the linear superposition of all the possible solutions with the same probability [67].

Step-2: Make $P_{T K V}(g)$ by observing $Q_{T K V}(g)$

Observe each Q-bit of $Q_{T K V}(g)$ to arrive at $P_{T K V}(g)$ where

$$
P_{T K V}(g)=\left\{p t k v_{1}^{g}, p t k v_{2}^{g}, \ldots, p t k v_{N}^{g}\right\}
$$

where $n$ is the total number of views in the lattice, $S M_{V i}$ is status materialized of view $V_{i}\left(S M_{V i}=1\right.$, if materialized, $S M_{V i}=0$, if not materialized), $\operatorname{Size}\left(V_{i}\right)$ is the size of view $V_{i}$ and $\operatorname{SizeSMA}\left(V_{i}\right)$ is the size of smallest materialized ancestor of view $V_{i}$

\section{Step-4: Create $B_{T K V}(g) / D_{T K V}(g)$ and $B_{T K V} / D_{T K V}$}

In this step, the initial best Top- $K$ views are selected, from among the binary Top- $K$ views in $P_{T K V}(g)$, and stored into $B_{T K V}$. Also, the best $T o p-K$ views among the equivalent decimal representation $D P_{T K V}(g)$ of $P_{T K V}(g)$ are selected and stored into $D_{T K V}$.

Initially, at $g=0$, store all $N$ Top- $K$ views in $P_{T K V}(g)$ and in corresponding $D P_{T K V}(g)$ into $B_{T K V}(g)$ and $D_{T K V}(g)$, respectively, where

$$
\begin{aligned}
& B_{T K V}(g)=\left\{b t k v_{1}^{g}, b t k v_{2}^{g}, \ldots, b t k v_{N}^{g}\right\} \\
& D_{T K V}(g)=\left\{d t k v_{1}^{g}, d t k v_{2}^{g}, \ldots, d t k v_{N}^{g}\right\}
\end{aligned}
$$

$$
p t k v_{i}^{g}=\left(\begin{array}{r}
\underbrace{p v_{i 11}^{g} p v_{i 12}^{g} \cdots p v_{i 1 d}^{g}}_{p v_{i 1}^{g}}|\underbrace{p v_{i 21}^{g} p v_{i 22}^{g} \cdots p v_{i 2 d}^{g}}_{p v_{i 2}^{g}}| \cdots \\
\forall i=1,2, \ldots, N ; j=1,2, \ldots, K ; l=1,2, \ldots, d .
\end{array}\right.
$$

$$
p v_{i j l}^{g}= \begin{cases}0, & \text { random }[0,1)<\left|\alpha v_{i j l}\right|^{2} \\ 1, & \text { otherwise }\end{cases}
$$

Each binary solution $p t k v_{i}^{g}$ is a binary string of length $K$, which is formed by selecting either 0 or 1 for each bit with the probability $\left|\alpha v_{i j l}\right|^{2}$.

\section{Step-3: Evaluate $P_{T K V}(g)$}

Each binary solution $P_{T K V}(g)$ is evaluated using the fitness function TVEC. Each Top-K view $p t k v_{i}^{g}$ in $P_{T K V}(g)$ is transformed into equivalent decimal representation $d t k v_{i}$ to arrive at $D P_{T K V}(g)$, where

$$
\begin{aligned}
D P_{T K V}(g) & =\left\{d p t k v_{1}^{g}, d p t k v_{2}^{g}, \ldots, d p t k v_{N}^{g}\right\} \text { and } \\
d p t k v_{i}^{g} & =\left\{d p v_{i 1}^{g}, d p v_{i 2}^{g}, \ldots, d p v_{i K}^{g}\right\}, i=1,2, \ldots N
\end{aligned}
$$

Compute TVEC of each view $d p t k v_{i}^{g}$ in $D P_{T K V}(g)$ as follows [64-66, 95-97]:

$$
\begin{aligned}
\operatorname{TVEC}\left(\operatorname{dptkv_{i}^{g})=}\right. & \sum_{i=1 \wedge S M_{V_{i}}=1}^{n} \operatorname{Size}\left(V_{i}\right) \\
& +\sum_{i=1 \wedge S M_{V_{i}}=0}^{n} \operatorname{SizeSMA}\left(V_{i}\right)
\end{aligned}
$$

$$
\begin{aligned}
& \text { for } i=1,2, \ldots, N \\
& b t k v_{i}^{g}=\left\{b v_{i 1}^{g}, b v_{i 2}^{g}, \ldots, b v_{i K}^{g}\right\} \\
& d t k v_{i}^{g}=\left\{d v_{i 1}^{g}, d v_{i 2}^{g}, \ldots, d v_{i K}^{g}\right\} \\
& \quad \text { and } \forall i=1,2, \ldots, N \\
& b t k v_{i}^{g}=p t k v_{i}^{g}, d t k v_{i}^{g}=d p t k v_{i}^{g} .
\end{aligned}
$$

The best Top- $K$ view in $B_{T K V}(g)$ and in the corresponding $D_{T K V}(g)$ are stored into $B_{T K V}$ and $D_{T K V}$, respectively. Increment $g$ by 1 .

\section{Step-5: Obtain $P_{T K V}(g)$ by observing $Q_{T K V}(g-1)$}

Binary solutions in $P_{T K V}(g)$ are formed by observing the states of $Q_{T K V}(g-1)$, as carried out in Step-2.

\section{Step-6: Evaluate $P_{T K V}(g)$}

Each binary solution $P_{T K V}(g)$ is evaluated using fitness function TVEC, as evaluated in Step-3.

\section{Step-7: Update $Q_{T K V}(g-1)$ using Q-gates}

Q-bit individuals in $Q_{T K V}(g)$ are updated by applying rotation Q-gate operator of QIEA [67] with the updated Qbit satisfying the normalization condition 


\begin{tabular}{|c|c|c|c|c|c|}
\hline \multirow{2}{*}{$\boldsymbol{x}$} & \multirow{2}{*}{$\boldsymbol{b}$} & \multirow{T}{*}{$\begin{array}{c}\text { TVEC(ptkv) } \\
\leq\end{array}$} & \multirow{2}{*}{$\Delta \theta_{i j l}^{g-1}$} & \multicolumn{2}{|c|}{$s\left(\alpha v_{i j l}^{g-1}, \beta v_{i j l}^{g-1}\right)$} \\
\cline { 5 - 6 } & & TVEC(btkv) & & $\alpha v_{i j l}^{g-1} \beta v_{i j l}^{g-1} \geq 0$ & $\alpha v_{i j l}^{g-1} \beta v_{i j l}^{g-1}<0$ \\
\hline 0 & 0 & false & 0 & \pm 1 & \pm 1 \\
\hline 0 & 0 & true & 0 & \pm 1 & \pm 1 \\
\hline 0 & 1 & false & $0.01 \pi$ & 1 & -1 \\
\hline 0 & 1 & true & 0 & \pm 1 & \pm 1 \\
\hline 1 & 0 & false & $0.01 \pi$ & -1 & 1 \\
\hline 1 & 0 & true & 0 & \pm 1 & \pm 1 \\
\hline 1 & 1 & false & 0 & \pm 1 & \pm 1 \\
\hline 1 & 1 & true & 0 & \pm 1 & \pm 1 \\
\hline
\end{tabular}

Figure 6. Look-up table [68].

$\left|\alpha v_{i j l}^{g}\right|^{2}+\left|\beta v_{i j l}^{g}\right|^{2}=1$, where $\alpha v_{i j l}^{g}$ and $\beta v_{i j l}^{g}$ are the values of the updated Q-bits.

$Q_{T K V}(g)$ is updated using quantum rotation gate as Qgate. The $l^{\text {th }} \mathrm{Q}$-bit of the $j^{\text {th }}$ view of the $i^{\text {th }} T o p-K$ view $q t k v_{i}^{g}, i=1,2, \ldots, N ; j=1,2, \ldots, K ; l=1,2, \ldots, d$ is updated by applying the current Q-gate $G_{i j l}^{g}(\theta)$ :

$$
G_{i j l}^{g-1}(\theta)=\left[\begin{array}{cc}
\cos \theta_{i j l}^{g-1} & -\sin \theta_{i j l}^{g-1} \\
\sin \theta_{i j l}^{g-1} & \cos \theta_{i j l}^{g-1}
\end{array}\right]
$$

where $\theta_{i j l}^{g}$ is an adjustable Q-gate rotation angle.

The Q-bit $\left[\alpha v_{i j l}^{g}, \beta v_{i j l}^{g}\right]$ is updated as follows:

$$
\left[\begin{array}{c}
\alpha v_{i j l}^{g} \\
\beta v_{i j l}^{g}
\end{array}\right]=G_{i j l}^{g-1}(\theta)\left[\begin{array}{c}
\alpha v_{i j l}^{g-1} \\
\beta v_{i j l}^{g-1}
\end{array}\right]
$$

where $\theta_{i j l}^{g-1}=s\left(\alpha v_{i j l}^{g-1}, \beta v_{i j l}^{g-1}\right) \Delta \theta_{i j l}^{g-1}$ and $s\left(\alpha v_{i j l}^{g-1}, \beta v_{i j l}^{g-1}\right)$ and $\Delta \theta_{i j l}^{g-1}$ are the sign and the magnitude of the rotation gate, respectively. The values of $s\left(\alpha v_{i j l}^{g-1}, \beta v_{i j l}^{g-1}\right)$ and $\Delta \theta_{i j l}^{g-1}$ are taken from the look-up table [93] given in figure 6; $x$ and $b$ are bits of $p t k v_{i}^{g}$ and $B_{T K V}$, respectively.

Step-8: Update $B_{T K V}(g) / D_{T K V}(g)$ and $B_{T K V} / D_{T K V}$

In this step, $B_{T K V}(g)$ and $D_{T K V}(g)$ are generated and $B_{T K V}$ and $D_{T K V}$ are updated.

The best $N$ Top- $K$ views among $P_{T K V}(g)$ and $B_{T K V}(g-1)$ are stored into $B_{T K V}(g)$.

The best $N$ Top- $K$ views among $D P_{T K V}(g)$ and $D_{T K V}(g-1)$ are stored into $D_{T K V}(g)$.

The best Top- $K$ views in $B_{T K V}(g)$ and in the corresponding $D_{T K V}(g)$ are stored into $B_{T K V}$ and $D_{T K V}$, respectively.

Increment $g$ by 1 .

\section{Step-9: Migration condition}

In this step, the global migration condition is checked. If it is satisfied, the best solution $B_{T K V}$ is migrated to $B_{T K V}(g)$ globally. Otherwise, the best Top- $K$ views in a local group in $B_{T K V}(g)$ are migrated to others in the same local group. Variation of probabilities of a Q-bit individual is induced during the migration process. The global migration and the local migration are carried out after a pre-specified number of generations $G_{G M}$ and $G_{L M}$, respectively.

\section{IF global migration THEN}

migrate $B_{T K V}$ to $B_{T K V}(g)$ globally

replace all the Top- $K$ views in $B_{T K V}(g)$ by $B_{T K V}$

\section{ELSE}

migrate $B_{T K V}$ to $B_{T K V}(g)$ locally

replacing the Top- $K$ views in each of the pre-specified number of local groups in $B(g)$ by best Top- $K$ views amongst them in the respective groups.

\section{Step-10: Termination condition}

QIEVSA runs for a pre-specified number of generations $G$, whereafter it produces the Top-K views $D_{T K V}$ as output.

IF $(g \leq G)$ THEN

\section{GO TO Step-5}

\section{ELSE}

Return $D_{T K V}$ as the Top- $K$ views.

Next, an example illustrating the use of QIEVSA to select Top- $K$ views from a multidimensional lattice is discussed.

\section{An example}

Consider the selection of Top-5 views from a three-dimensional lattice shown in figure 3 using QIEVSA.

\section{Step-1: Initialization}

Generation, $g=0 ;$ quantum population size, $\quad N=10$; number of Q-bits in quantum gene, $d=3$. Since Top -5 views need to be selected, the quantum chromosome length $K=5$. The quantum population of Top- $K$ views at $g=0$, i.e., $Q_{T 5 v}(0)=\left\{q t 5 v_{1}^{0}, q t 5 v_{2}^{0}, \ldots, q t 5 v_{10}^{0}\right\}$ is presented in figure 7. Initially, $\alpha v_{i k l}^{O}$ and $\beta v_{i k l}^{o}$ are taken as $1 /$ $\sqrt{ } 2(0.7071)$.

\section{Step-2: Make $P_{T 5 V}(0)$ by observing $Q_{T 5 V}(0)$}

Quantum observed state $P_{T 5 V}(0)=\left\{p t 5 v_{1}^{0}, p t 5 v_{2}^{0}, \ldots\right.$, $\left.p t 5 v_{10}^{0}\right\}$ in binary form, i.e., Q-bit representation of initial population $Q_{T 5 V}(0)$ is observed. The observation of the first Top-5 views $q t 5 v_{1}^{0}$ in $Q_{T 5 V}(0)$ to generate $p t 5 v_{1}^{0}$ is shown in figure 8 .

In a similar manner, quantum observed state for other Top- $K$ views in $Q_{T 5 V}(0)$ are computed to generate $P_{T 5 V}(0)$, which is given in figure 9 .

\section{Step-3: Evaluate $P_{T 5 V}(0)$}

Each Top-5 views $p t 5 v_{i}^{0}(i=0,1, \ldots, 10)$ in $P_{T 5 V}(0)$ is transformed into equivalent decimal representation, i.e., $d t 5 v_{\mathrm{i}}^{0}$ to generate $D P_{T 5 v}(0)=\left\{d p t 5 v_{1}^{0}, d p t 5 v_{1}^{0}, \ldots, d p t 5 v_{10}^{0}\right\}$, 


\begin{tabular}{|c|c|c|c|c|c|c|c|c|c|c|c|c|c|c|c|}
\hline \multirow{2}{*}{$Q_{T S V}(0)$} & $v_{i 11}^{0}$ & $\alpha v_{i 12}^{0}$ & $\alpha v_{i 13}{ }^{0}$ & $\alpha v_{i 21}^{0}$ & $\alpha v_{i 22}{ }^{0}$ & $\alpha v_{i 23}{ }^{0}$ & $\alpha v_{i 31}{ }^{0}$ & $\alpha v_{i 32}{ }^{0}$ & $\alpha v_{i 33}{ }^{0}$ & $\alpha v_{i 41}{ }^{0}$ & $\alpha v_{i 42}^{0}$ & $\alpha v_{i 43}{ }^{0}$ & $\alpha v_{i 51}{ }^{0}$ & $\alpha v_{i 52}{ }^{0}$ & $\alpha v_{i 53}{ }^{0}$ \\
\hline & $\beta v_{i 11}^{0}$ & $\beta v_{i 12}^{0}$ & $\beta v_{i 13}{ }^{0}$ & $v_{i 21}^{0}$ & $\beta v_{i 22}^{0}$ & $p v_{i 23}$ & $\beta v_{i 31}^{0}$ & $\beta v_{i 32}{ }^{0}$ & $\beta v_{i 33}{ }^{0}$ & $p v_{i 41}$ & $\beta v_{i 42}{ }^{0}$ & $\beta v_{i 43}{ }^{0}$ & $\beta v_{i 51}{ }^{0}$ & $\beta v_{i 52}{ }^{0}$ & $v_{i 53}{ }^{0}$ \\
\hline \multirow{2}{*}{$q t 5 v_{1}{ }^{0}$} & 071 & 0.7071 & 0.7071 & 071 & 071 & 0.7071 & 0.7071 & 7071 & 7071 & 0.7071 & 0.7071 & 7071 & 0.7071 & 7071 & 7071 \\
\hline & & & & & & & & & & & & & & & 7071 \\
\hline \multirow{2}{*}{$q t 5 v_{2}^{0}$} & 071 & & & 0.7071 & 0.70 & 0.7071 & & & & & & & & & 7071 \\
\hline & 0.7071 & 0.7071 & 0.70 & 0.7071 & 0 & 0.7071 & 0.7071 & 0 & 71 & 0.7071 & & & 0. & & 7071 \\
\hline \multirow{2}{*}{$q t 5 v_{3}^{0}$} & \begin{tabular}{|l|}
0.7071 \\
\end{tabular} & 0.7071 & 0.7071 & 0.7071 & $0.70^{\prime}$ & 0.7071 & \begin{tabular}{|l|}
0.7071 \\
\end{tabular} & 71 & 0.7071 & 0. & 71 & 71 & 0.7 & & 071 \\
\hline & 10 & & & 0. & & & & & & & & & & & 011 \\
\hline \multirow{2}{*}{$q t 5 v_{4}{ }^{0}$} & 0.7071 & & & \begin{tabular}{|l|}
0.7071 \\
\end{tabular} & 0 . & 0.7 & & & 0.7 & & & & & & 071 \\
\hline & 0.7071 & 0.7071 & 0.7 & 0.7071 & 0 & 0.7071 & 0.7 & 0. & 71 & 0.7071 & & & 0.7 & & 7071 \\
\hline \multirow{2}{*}{$q t 5 v_{5}^{0}$} & 0.7071 & 0.7071 & 0.7071 & 0.7071 & 0.7071 & 0.7071 & 0.7 & 0.7 & 0.7 & 0.7 & 0 & 71 & 0.7 & & 071 \\
\hline & 7071 & & & 0.7 & 0 & 0. & & & 0 & & & & & & 971 \\
\hline \multirow{2}{*}{$q t 5 v_{6}^{0}$} & 0.707 & 0.70 & 0.7 & 0.7071 & 0.7 & 0.70 & 0.70 & 0.7 & 0.7071 & \begin{tabular}{|l|l|} 
\\
\end{tabular} & & & 0.7 & & 7071 \\
\hline & 0.7071 & 0.7 & & 0. & 0.7 & 0.7 & & & 0. & 0.7 & & & 0.7 & & .7071 \\
\hline \multirow{2}{*}{$q t 5 v_{7}^{0}$} & 0.7071 & 0.7 & 0.7 & \begin{tabular}{|l|l|}
0.7071 \\
\end{tabular} & 0.70 & 0.7 & 0.7 & 0. & 0. & 0.7 & 0 & 71 & 0.7 & & 0.7071 \\
\hline & 0.7071 & 0.7071 & 0.7 & 0.7071 & 0.70 & 0.7071 & 0.7071 & 0.7 & 0.7071 & 0.70 & & 71 & 0.7 & & 0.7071 \\
\hline \multirow{2}{*}{$q t 5 v_{8}^{0}$} & .7071 & 0.7071 & 0.7071 & \begin{tabular}{|l|l|}
0.7071 \\
\end{tabular} & 0.70 & 0.7071 & 0.7071 & 0.7071 & 0.7071 & 0.70 & 0. & 71 & 0.7 & & 7071 \\
\hline & 0.7071 & & & & & & & & & & & & & & 071 \\
\hline \multirow{2}{*}{$q t 5 v_{9}^{0}$} & 0.7071 & 0.7071 & 0.7071 & 0.7071 & 0.7071 & 0.7071 & 0.7071 & 0.7071 & 0.7071 & 0.7071 & 0.7071 & 0.7071 & 0.7071 & & 0.7071 \\
\hline & 0.7071 & 0.7 & 0.7 & 0.7071 & 0.7 & 0.7 & 0.7 & 0. & 0.7 & 0.7 & & & 0.7 & & 0.7071 \\
\hline \multirow{2}{*}{$q t 5 v_{10}{ }^{0}$} & 0.7071 & 0.7071 & 0.70 & 0.7071 & 0.70 & 0.7071 & 0.7071 & 0.7 & 0.7071 & 0.7 & & & 0.7 & & 7071 \\
\hline & 707 & & & .7071 & & & & & & & & 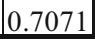 & & & 0.707 \\
\hline
\end{tabular}

Figure 7. $Q_{T S V}(0)$.

\begin{tabular}{|c|c|c|c|}
\hline Random var. & $\alpha v_{1 j l^{2}}^{0}$ & $p v_{1 j l}^{0}$ & $p t 5 v_{i}^{0}$ \\
\hline 0.40175587 & 0.49999997 & 0 & \multirow{3}{*}{011} \\
\hline 0.51350707 & 0.49999997 & 1 & \\
\hline 0.6879447 & 0.49999997 & 1 & \\
\hline 0.4345283 & 0.49999997 & 0 & \multirow{3}{*}{001} \\
\hline 0.34838796 & 0.49999997 & 0 & \\
\hline 0.7124095 & 0.49999997 & 1 & \\
\hline 0.7756701 & 0.49999997 & 1 & \multirow{3}{*}{101} \\
\hline 0.30042487 & 0.49999997 & 0 & \\
\hline 0.9100001 & 0.49999997 & 1 & \\
\hline 0.78266287 & 0.49999997 & 1 & \multirow{3}{*}{110} \\
\hline 0.9099875 & 0.49999997 & 1 & \\
\hline 0.000876069 & 0.49999997 & 0 & \\
\hline 0.58374248 & 0.49999997 & 1 & \multirow{3}{*}{111} \\
\hline 0.56351848 & 0.49999997 & 1 & \\
\hline 0.69518647 & 0.49999997 & 1 & \\
\hline
\end{tabular}

Figure 8. $p t 5 v_{1}^{0}$ of $q t 5 v_{1}^{0}$ in $Q_{T S V}(0)$.

where $d p t 5 v_{i}^{0}=\left\{d p v_{i 1}^{0}, d p v_{1}^{0}, \ldots, d p v_{5}^{0}\right\} . D P_{T 5 V}(0)$ is shown in figure 10. TVEC of each view $d p t 5 v_{i}^{0}$ in $D P_{T 5 V}(0)$ is computed. TVEC computation of $d p t 5 v_{1}^{0}$ comprising views $C T, C P, P, T$ and None is shown in figure 11. In a similar manner, $T V E C$ of other $d p t 5 v_{i}^{0}$ in $D P_{T 5 V}(0)$ are computed and are shown in figure 12 .

\section{Step-4: Create $B_{T 5 V}(0)$ and $B_{T 5 V}$}

$P_{T 5 V}(0)$ and $D P_{T 5 V}(0)$ are stored into $B_{T 5 V}(0)$ and $D_{T 5 V}(0)$, respectively. $B_{T 5 V}(0)$ and $D_{T 5 V}(0)$ are shown in figures 13 and 14, respectively.
The best Top-5 views in $B_{T 5 V}(0)$ and $D_{T 5 V}(0)$ are stored into $B_{T 5 V}$ and $D_{T 5 V}$, respectively. They are shown in figure 15. Next, $g$ is incremented by 1, i.e., $g=1+1=2$.

\section{Step-5: Make $P_{T 5 V}(1)$ by observing $Q_{T 5 V}(0)$}

Quantum observed state $P_{T 5 V}(1)=\left\{p t 5 v_{1}^{1}, p t 5 v_{2}^{1}, \ldots\right.$, $\left.p t 5 v_{10}^{1}\right\}$ in binary form is generated. The observation of the first $T o p-5$ views $q t 5 v_{1}^{0}$ in $Q_{T 5 V}(0)$ to generate $p t 5 v_{1}^{1}$ is shown in figure 16.

In a similar manner, quantum observed states for other Top- $K$ views in $Q_{T 5 V}(0)$ are computed to generate $P_{T 5 V}(1)$, which is given in figure 17.

\section{Step-6: Evaluate $P_{T 5 V}(1)$}

Each Top-5 views $p t 5 v_{i}^{O}(i=0,1, \ldots, 10)$ in $P_{T 5 V}(1)$ is transformed into equivalent decimal representation, i.e., $d t 5 v_{i}^{0}$, to generate $D P_{T 5 V}(1)=\left\{d p t 5 v_{1}^{1}, d p t 5 v_{1}^{1}, \ldots, d p t 5 v_{10}^{1}\right\}$, where $d p t 5 v_{i}^{1}=\left\{d p v_{i 1}^{1}, d p v_{1}^{1}, \ldots, d p v_{5}^{1}\right\} . D P_{T 5 V}(1)$ is shown in figure 18. TVEC of each view $d p t 5 v_{i}^{1}$ in $D P_{T K V}(1)$ is computed and are shown in figure 19.

\section{Step-7: Update $Q_{T 5 V}(0)$ using $Q$-gates}

Update Q-bits $\left(\alpha v_{i k l}^{0}\right.$ and $\left.\beta v_{i k l}^{0}\right)$ of each Top-5 views in $Q_{T 5 V}(0)$ using corresponding Top-5 views in $P_{T 5 V}(1)$ and best Top-5 views $B_{T 5 V}$. Updation of Q-bit $\left(\alpha v_{l k l}^{0}\right.$ and $\left.\beta v_{l k l}^{0}\right)$ of the first individual $q t 5 v_{1}^{0}$ of $Q_{T K V}(0)$ using $p t 5 v_{1}^{1}$, in $P_{T 5 V}(1)$, and $B_{T 5 V}$, shown in figure 20 , is given below:

\section{Computation of $\theta_{111}^{\mathbf{0}}$ for the first bit}

Bit $x=1$, bit $b=1, \operatorname{TVEC}\left(p t 5 v_{1}^{1}\right)=187$ and TVEC $\left(B_{T 5 V}\right)$ $=181$. Since TVEC $\left(p t 5 v_{1}^{1}\right) \leq T V E C\left(B_{T 5 V}\right)$ is false, using the look-up table, $s\left(\alpha v_{111}^{0}, \beta v_{111}^{0}\right)=1$ and $\Delta \theta_{111}^{0}=0$. 


\begin{tabular}{|c|c|c|c|c|c|}
\hline$P_{T S V}(0)$ & $p v_{i 1}^{0}$ & $p v_{i 2}^{0}$ & $p v_{i 3}^{0}$ & $p v_{i 4}^{0}$ & $p v_{i 5}^{0}$ \\
\hline$p t 5 v_{1}{ }^{0}$ & 011 & 001 & 101 & 110 & 111 \\
\hline$p t 5 v_{2}{ }^{0}$ & 001 & 011 & 110 & 111 & 101 \\
\hline$p t 5 v_{3}{ }^{0}$ & 001 & 110 & 100 & 101 & 000 \\
\hline$p t 5 v_{4}{ }^{0}$ & 011 & 110 & 001 & 101 & 111 \\
\hline$p t 5 v_{5}{ }^{0}$ & 110 & 111 & 011 & 101 & 010 \\
\hline$p t 5 v_{6}{ }^{0}$ & 100 & 111 & 101 & 110 & 011 \\
\hline$p t 5 v_{7}^{0}$ & 101 & 100 & 001 & 011 & 111 \\
\hline$p t 5 v_{8}{ }^{0}$ & 001 & 011 & 010 & 101 & 111 \\
\hline$p t 5 v_{9}{ }^{0}$ & 111 & 010 & 100 & 101 & 011 \\
\hline$p t 5 v_{10}{ }^{0}$ & 101 & 110 & 100 & 001 & 111 \\
\hline
\end{tabular}

Figure 9. $P_{T 5 V}(0)$.

\begin{tabular}{|c|c|c|c|c|c|c|}
\hline$P_{T S V}(0)$ & $D_{T S V}(0)$ & $d p v_{i 1}^{0}$ & $d p v_{i 2}^{0}$ & $d p v_{i 3}^{0}$ & $d p v_{i 4}^{0}$ & $d p v_{i 5}^{0}$ \\
\hline$p t 5 v_{1}^{0}$ & $d p t 5 v_{1}^{0}$ & 3 & 1 & 5 & 6 & 7 \\
\hline$p t 5 v_{2}^{0}$ & $d p t 5 v_{2}{ }^{\circ}$ & 1 & 3 & 6 & 7 & 5 \\
\hline$p t 5 v_{3}{ }^{0}$ & $d p t 5 v_{3}^{0}$ & 1 & 6 & 4 & 5 & 7 \\
\hline$p t 5 v_{4}^{0}$ & $d p t 5 v_{4}{ }^{0}$ & 3 & 6 & 1 & 5 & 7 \\
\hline$p t 5 v_{5}^{0}$ & $d p t 5 v_{5}^{0}$ & 6 & 7 & 3 & 5 & 2 \\
\hline$p t 5 v_{6}^{0}$ & $d p t 5 v_{6}^{0}$ & 4 & 7 & 5 & 6 & 3 \\
\hline$p t 5 v_{7}^{0}$ & $d p t 5 v_{7}^{0}$ & 5 & 4 & 1 & 3 & 7 \\
\hline$p t 5 v_{8}^{0}$ & dpt5v $v_{8}^{0}$ & 1 & 3 & 2 & 5 & 7 \\
\hline$p t 5 v_{9}{ }^{0}$ & $d p t 5 v_{9}^{0}$ & 7 & 2 & 4 & 5 & 3 \\
\hline$p t 5 v_{10}{ }^{\circ}$ & $d p t 5 v_{10}{ }^{0}$ & 5 & 6 & 4 & 1 & 7 \\
\hline
\end{tabular}

Figure 10. $D P_{T 5 V}(0)$.

$$
\theta_{111}^{0}=s\left(\alpha v_{111}^{0}, \beta v_{111}^{0}\right) \Delta \theta_{111}^{0}=1 \times 0=0 .
$$

\section{Computation of $\theta_{112}^{0}$ for the second bit}

Bit $x=1$, bit $b=1, \operatorname{TVEC}\left(p t 5 v_{1}^{1}\right)=187$ and $\operatorname{TVEC}\left(B_{T 5 V}\right)$ $=181$. Since $\operatorname{TVEC}\left(p t 5 v_{1}^{1}\right) \leq \operatorname{TVEC}\left(B_{T 5 V}\right)$ is false, using the look-up table, $s\left(\alpha v_{112}^{0}, \beta v_{112}^{0}\right)=1$ and $\Delta \theta_{112}^{0}=0$.

$$
\theta_{112}^{0}=s\left(\alpha v_{112}^{0}, \beta v_{112}^{0}\right) \Delta \theta_{112}^{0}=1 \times 0=0 .
$$

\section{Computation of $\theta_{113}^{0}$ for the third bit}

Bit $x=0$, bit $b=1, \operatorname{TVEC}\left(p t 5 v_{1}^{1}\right)=187$ and $\operatorname{TVEC}\left(B_{T 5 V}\right)$ $=181$. Since $\operatorname{TVEC}\left(p t 5 v_{1}^{1}\right) \leq \operatorname{TVEC}\left(B_{T 5 V}\right)$ is false, using the look-up table, $s\left(\alpha v_{113}^{0}, \beta v_{113}^{0}\right)=1$ and $\Delta \theta_{113}^{0}=0.0314$.

$$
\theta_{113}^{0}=s\left(\alpha v_{113}^{0}, \beta v_{113}^{0}\right) \Delta \theta_{113}^{0}=1 \times 0.0314=0.0314
$$

In a similar manner, $\theta_{1 \mathrm{kl}}^{0}$ for the other quantum bits of the first individual $q t 5 v_{1}^{0}$ of $Q_{T 5 v}(0)$ is computed and is shown in figure 21 .

\begin{tabular}{|c|c|c|}
\hline$P_{T 5 V}(0)$ & $D_{T 5 V}(0)$ & TVEC \\
\hline$p t 5 v_{1}{ }^{0}$ & $d p t 5 v_{1}{ }^{0}$ & 187 \\
\hline$p t 5 v_{2}{ }^{0}$ & $d p t 5 v_{2}{ }^{0}$ & 187 \\
\hline$p t 5 v_{3}{ }^{0}$ & $d p t 5 v_{3}{ }^{0}$ & 183 \\
\hline$p t 5 v_{4}{ }^{0}$ & $d p t 5 v_{4}{ }^{0}$ & 187 \\
\hline$p t 5 v_{5}{ }^{0}$ & $d p t 5 v_{5}{ }^{0}$ & 187 \\
\hline$p t 5 v_{6}{ }^{0}$ & $d p t 5 v_{6}{ }^{0}$ & 185 \\
\hline$p t 5 v_{7}{ }^{0}$ & $d p t 5 v_{7}{ }^{0}$ & 187 \\
\hline$p t 5 v_{8}^{0}$ & $d p t 5 v_{8}{ }^{0}$ & 183 \\
\hline$p t 5 v_{9}{ }^{0}$ & $d p t 5 v_{9}{ }^{0}$ & 181 \\
\hline$p t 5 v_{10}{ }^{0}$ & $d p t 5 v_{10}{ }^{0}$ & 183 \\
\hline
\end{tabular}

Figure 12. TVEC of $d p t 5 v_{i}^{0}$ in $D P_{T 5 V}(0)$.

\begin{tabular}{|c|c|c|c|c|c|}
\hline$B_{T 5 V}(0)$ & $b v_{i 1}^{0}$ & $b v_{i 2}^{0}$ & $b v_{i 3}^{0}$ & $b v_{i 4}^{0}$ & $b v_{i 5}^{0}$ \\
\hline$b t 5 v_{1}{ }^{0}$ & 011 & 001 & 101 & 110 & 111 \\
\hline$b t 5 v_{2}{ }^{0}$ & 001 & 011 & 110 & 111 & 101 \\
\hline$b t 5 v_{3}{ }^{0}$ & 001 & 110 & 100 & 101 & 000 \\
\hline$b t 5 v_{4}{ }^{0}$ & 011 & 110 & 001 & 101 & 111 \\
\hline$b t 5 v_{5}{ }^{0}$ & 110 & 111 & 011 & 101 & 010 \\
\hline$b t 5 v_{6}{ }^{0}$ & 100 & 111 & 101 & 110 & 011 \\
\hline$b t 5 v_{7}{ }^{0}$ & 101 & 100 & 001 & 011 & 111 \\
\hline$b t 5 v_{8}{ }^{0}$ & 001 & 011 & 010 & 101 & 111 \\
\hline$b t 5 v_{9}{ }^{0}$ & 111 & 010 & 100 & 101 & 011 \\
\hline$b t 5 v_{10}{ }^{0}$ & 101 & 110 & 100 & 001 & 111 \\
\hline
\end{tabular}

Figure 13. $B_{T 5 V}(0)$.

\begin{tabular}{|c|c|c|c|c|c|}
\hline$D_{T 5 V}(0)$ & $d v_{i 1}^{0}$ & $d v_{i 2}^{0}$ & $d v_{i 3}^{0}$ & $d v_{i 4}^{0}$ & $d v_{i 5}^{0}$ \\
\hline$d t 5 v_{1}^{0}$ & 3 & 1 & 5 & 6 & 7 \\
\hline$d t 5 v_{2}{ }^{0}$ & 1 & 3 & 6 & 7 & 5 \\
\hline$d t 5 v_{3}{ }^{0}$ & 1 & 6 & 4 & 5 & 7 \\
\hline$d t 5 v_{4}{ }^{0}$ & 3 & 6 & 1 & 5 & 7 \\
\hline$d t 5 v_{5}{ }^{0}$ & 6 & 7 & 3 & 5 & 2 \\
\hline$d t 5 v_{6}{ }^{0}$ & 4 & 7 & 5 & 6 & 3 \\
\hline$d t 5 v_{7}{ }^{0}$ & 5 & 4 & 1 & 3 & 7 \\
\hline$d t 5 v_{8}^{0}$ & 1 & 3 & 2 & 5 & 7 \\
\hline$d t 5 v_{9}{ }^{0}$ & 7 & 2 & 4 & 5 & 3 \\
\hline$d t 5 v_{10}{ }^{0}$ & 5 & 6 & 4 & 1 & 7 \\
\hline
\end{tabular}

Figure 14. $D_{T 5 V}(0)$.

$$
\begin{aligned}
& \sum_{i=1 \wedge S M_{V_{i}}=1}^{8} \operatorname{Size}\left(\operatorname{dpt} 5 v_{1}{ }^{0}\right)=(\operatorname{Size}(C P T)+\operatorname{Size}(C T)+\operatorname{Size}(C P)+\operatorname{Size}(P)+\operatorname{Size}(T)+\operatorname{Size}(\text { None })) \\
& =(40+28+26+14+12+1)=121, \\
& \sum_{i=1 \wedge S M_{V_{i}}=0}^{8} \operatorname{SizeSMA}\left(\operatorname{dpt} 5 v_{1}{ }^{0}\right)=(\operatorname{SizeSMA}(P T)+\operatorname{SizeSMA}(C))=(40+26)=66 \\
& \text { TVEC }=\sum_{i=1 \wedge S M_{V}=1}^{8} \operatorname{Size}\left(\operatorname{dpt} 5 v_{1}{ }^{0}\right)+\sum_{i=1 \wedge S M_{V}=0}^{8} \operatorname{SizeSMA}\left(\operatorname{dpt} 5 v_{1}{ }^{0}\right)=121+66=187 .
\end{aligned}
$$

Figure 11. TVEC computation if views $C T, C P, P, T$ and None are selected for materialization. 


\begin{tabular}{|c|c|c|c|c|c|c|}
\hline Top-5 views & View-1 & View-2 & View-3 & View-4 & View-5 & TVEC \\
\hline$B_{T S V}$ & 111 & 010 & 100 & 101 & 011 & 181 \\
\hline$D_{T S V}$ & 7 & 2 & 4 & 5 & 3 & 181 \\
\hline
\end{tabular}

Figure 15. $B_{T 5 V}$ and $D_{T 5 V}$.

\begin{tabular}{|c|c|c|c|}
\hline Random $[0,1)$ & $\alpha_{1 j l}^{1}$ & $p v_{1 j l}^{1}$ & $p t 5 v_{i}^{1}$ \\
\hline 0.5011488 & 0.499999997 & 1 & \multirow{3}{*}{110} \\
\hline 0.70176893 & 0.499999997 & 1 & \\
\hline 0.043875396 & 0.49999997 & 0 & \\
\hline 0.73296607 & 0.499999997 & 1 & \multirow{3}{*}{101} \\
\hline 0.1709851 & 0.49999997 & 0 & \\
\hline 0.93461823 & 0.499999997 & 1 & \\
\hline 0.9480489 & 0.499999997 & 1 & \multirow{3}{*}{111} \\
\hline 0.6981055 & 0.49999997 & 1 & \\
\hline 0.7665917 & 0.499999997 & 1 & \\
\hline 0.02548498 & 0.499999997 & 0 & \multirow{3}{*}{011} \\
\hline 0.51505903 & 0.499999997 & 1 & \\
\hline 0.64097357 & 0.499999997 & 1 & \\
\hline 0.4138329 & 0.49999997 & 0 & \multirow{3}{*}{010} \\
\hline 0.9327696 & 0.49999997 & 1 & \\
\hline 0.0682677 & 0.49999997 & 0 & \\
\hline
\end{tabular}

Figure 16. $p t 5 v_{1}^{1}$ of $q t 5 v_{1}^{0}$ in $Q_{T 5 V}(0)$.

Updation of $\alpha v_{111}^{0}$ and $\beta v_{111}^{0}$ of the first Q-bit $\left[\begin{array}{l}\alpha v_{111}^{1} \\ \beta v_{111}^{1}\end{array}\right]=$ $\left[\begin{array}{cc}\cos 0 & -\sin 0 \\ \sin 0 & \cos 0\end{array}\right] \times\left[\begin{array}{l}0.70710677 \\ 0.70710677\end{array}\right]=\left[\begin{array}{ll}1 & 0 \\ 0 & 1\end{array}\right] \times$ $\left[\begin{array}{l}0.70710677 \\ 0.70710677\end{array}\right]=\left[\begin{array}{l}0.70710677 \\ 0.70710677\end{array}\right]$.

Updation of $\alpha v_{112}^{0}$ and $\beta v_{112}^{0}$ of the second Q-bit

$$
\begin{aligned}
{\left[\begin{array}{l}
\alpha v_{112}^{1} \\
\beta v_{112}^{1}
\end{array}\right] } & =\left[\begin{array}{cc}
\cos 0 & -\sin 0 \\
\sin 0 & \cos 0
\end{array}\right] \times\left[\begin{array}{l}
0.70710677 \\
0.70710677
\end{array}\right] \\
& =\left[\begin{array}{ll}
1 & 0 \\
0 & 1
\end{array}\right] \times\left[\begin{array}{l}
0.70710677 \\
0.70710677
\end{array}\right]=\left[\begin{array}{l}
0.70710677 \\
0.70710677
\end{array}\right] .
\end{aligned}
$$

Updation of $\alpha v_{113}^{0}$ and $\beta v_{113}^{0}$ of the third Q-bit

$$
\begin{aligned}
{\left[\begin{array}{l}
\alpha v_{113}^{1} \\
\beta v_{113}^{1}
\end{array}\right]=} & {\left[\begin{array}{cc}
\cos 0.0314 & -\sin 0.0314 \\
\sin 0.0314 & \cos 0.0314
\end{array}\right] } \\
& \times\left[\begin{array}{cc}
0.70710677 \\
0.70710677
\end{array}\right] \\
= & {\left[\begin{array}{cc}
0.9995 & -0.0314 \\
0.0314 & 0.9995
\end{array}\right] \times\left[\begin{array}{l}
0.70710677 \\
0.70710677
\end{array}\right] } \\
= & {\left[\begin{array}{l}
0.6846 \\
0.7290
\end{array}\right] . }
\end{aligned}
$$

In a similar manner, Q-bits of each $q t 5 v_{i}^{0}$ of $Q_{T 5 V}(0)$ are updated to generate $Q_{T 5 V}(1)$, which is shown in figure 22 .

\section{Step-8: Update $B_{T 5 V}(1) / D_{T 5 V}(1)$ and $B_{T 5 V} / D_{T 5 V}$}

The 10 Top -5 views, from amongst $P_{T 5 V}(1)$ and $B_{T 5 V}(0)$, are stored into $B_{T 5 V}(1)$. The 10 Top-5 views, from amongst

\begin{tabular}{|c|c|c|c|c|c|}
\hline$P_{T K V}(1)$ & $p v_{i 1}^{1}$ & $p v_{i 2}^{1}$ & $p v_{i 3}^{1}$ & $p v_{i 4}^{1}$ & $p v_{i 5}^{1}$ \\
\hline$p t 5 v_{1}{ }^{1}$ & 110 & 101 & 111 & 011 & 010 \\
\hline$p t 5 v_{2}{ }^{1}$ & 001 & 011 & 110 & 101 & 111 \\
\hline$p t 5 v_{3}{ }^{1}$ & 111 & 011 & 110 & 001 & 101 \\
\hline$p t 5 v_{4}{ }^{1}$ & 101 & 110 & 010 & 111 & 001 \\
\hline$p t 5 v_{5}{ }^{1}$ & 001 & 111 & 100 & 110 & 101 \\
\hline$p t 5 v_{6}{ }^{1}$ & 110 & 010 & 011 & 111 & 101 \\
\hline$p t 5 v_{7}{ }^{1}$ & 001 & 011 & 110 & 010 & 100 \\
\hline$p t 5 v_{8}{ }^{1}$ & 010 & 101 & 001 & 111 & 110 \\
\hline$p t 5 v_{9}{ }^{1}$ & 001 & 100 & 111 & 101 & 110 \\
\hline$p t 5 v_{10}{ }^{1}$ & 111 & 001 & 011 & 101 & 010 \\
\hline
\end{tabular}

Figure 17. $P_{T 5 V}(1)$.

\begin{tabular}{|c|c|c|c|c|c|c|}
\hline$P_{T 5 V}(1)$ & $D_{T 5 V}(1)$ & $d p v_{i 1}^{1}$ & $d p v_{i 2}^{1}$ & $d p v_{i 3}^{1}$ & $d p v_{i 4}^{1}$ & $d p v_{i 5}^{1}$ \\
\hline$p t 5 v_{1}{ }^{1}$ & $d p t 5 v_{1}{ }^{1}$ & 6 & 5 & 7 & 3 & 2 \\
\hline$p t 5 v_{2}^{1}$ & $d p t 5 v_{2}{ }^{1}$ & 1 & 3 & 6 & 5 & 7 \\
\hline$p t 5 v_{3}{ }^{1}$ & $d p t 5 v_{3}{ }^{1}$ & 7 & 3 & 6 & 1 & 5 \\
\hline$p t 5 v_{4}{ }^{1}$ & $d p t 5 v_{4}{ }^{1}$ & 5 & 6 & 2 & 7 & 1 \\
\hline$p t 5 v_{5}{ }^{1}$ & $d p t 5 v_{5}{ }^{1}$ & 1 & 7 & 4 & 6 & 5 \\
\hline$p t 5 v_{6}{ }^{1}$ & $d p t 5 v_{6}{ }^{1}$ & 6 & 2 & 3 & 7 & 5 \\
\hline$p t 5 v_{7}{ }^{1}$ & $d p t 5 v_{7}{ }^{1}$ & 1 & 3 & 6 & 2 & 4 \\
\hline$p t 5 v_{8}{ }^{1}$ & $d p t 5 v_{8}{ }^{1}$ & 2 & 5 & 1 & 7 & 6 \\
\hline$p t 5 v_{9}{ }^{1}$ & $d p t 5 v_{9}{ }^{1}$ & 1 & 4 & 7 & 5 & 6 \\
\hline$p t 5 v_{10}{ }^{1}$ & $d p t 5 v_{10}{ }^{1}$ & 7 & 1 & 3 & 5 & 2 \\
\hline
\end{tabular}

Figure 18. $D P_{T 5 V}(1)$.

\begin{tabular}{|c|c|c|}
\hline$P_{T 5 V}(1)$ & $D_{T 5 V}(1)$ & TVEC \\
\hline$p t 5 v_{1}{ }^{1}$ & $d p t 5 v_{1}{ }^{1}$ & 187 \\
\hline$p t 5 v_{2}{ }^{1}$ & $d p t 5 v_{2}{ }^{1}$ & 187 \\
\hline$p t 5 v_{3}{ }^{1}$ & $d p t 5 v_{3}{ }^{1}$ & 187 \\
\hline$p t 5 v_{v^{1}}{ }^{1}$ & $d p t 5 v_{4}{ }^{1}$ & 183 \\
\hline$p t 5 v_{5}{ }^{1}$ & $d p t 5 v_{5}{ }^{1}$ & 183 \\
\hline$p t 5 v_{6}{ }^{1}$ & $d p t 5 v_{6}{ }^{1}$ & 187 \\
\hline$p t 5 v_{7}{ }^{1}$ & $d p t 5 v_{7}{ }^{1}$ & 174 \\
\hline$p t 5 v_{8}{ }^{1}$ & $d p t 5 v_{8}{ }^{1}$ & 183 \\
\hline$p t 5 v_{9}{ }^{1}$ & $d p t 5 v_{9}{ }^{1}$ & 183 \\
\hline$p t 5 v_{10}{ }^{1}$ & $d p t 5 v_{10}{ }^{1}$ & 183 \\
\hline
\end{tabular}

Figure 19. TVEC of $d p t 5 v_{i}^{1}$ in $D P_{T 5 V}(1)$.

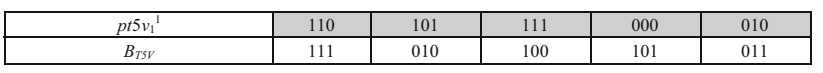

Figure 20. $p t 5 v_{1}^{1}$ and $B_{T 5 V}$.

$D P_{T 5 V}(1)$ and $D_{T 5 V}(0)$, are stored into $D_{T 5 V}(1) . B_{T 5 V}(1)$ and $D_{T 5 V}(1)$ are shown in figures 23 and 24, respectively. The best $T o p-K$ views in $B_{T 5 V}(1)$, and in the corresponding $D_{T 5 V}(1)$, are stored into $B_{T 5 V}$ and $D_{T 5 V}$, respectively. The updated $B_{T 5 V}$ and $D_{T 5 V}$ are shown in figure 25. This is followed by incrementing $g$ by 1 .

\section{Step-9: Perform local/global migration}

Considering that local migration is performed in each step, the resultant $D_{T 5 V}(1)$, after performing local migration for local group size 2 , is given in figure 26. 


\begin{tabular}{|c|c|c|c|c|c|c|c|c|}
\hline Bit & $x$ & $b$ & $T V E C\left(p t 5 v_{1}^{1}\right)$ & $T V E C\left(B_{T 5 V}\right)$ & $\begin{array}{c}\operatorname{TVEC}\left(p t 5 v_{1}\right) \\
\leq \\
T V E C\left(B_{T 5 V}\right)\end{array}$ & $\Delta \theta_{1 j l}^{0}$ & Sign & $\theta_{1 j l}^{0}$ \\
\hline 1 & 1 & 1 & 187 & 181 & False & 0 & 1 & 0 \\
\hline 2 & 1 & 1 & 187 & 181 & False & 0 & 1 & 0 \\
\hline 3 & 0 & 1 & 187 & 181 & False & 0.0314 & 1 & 0.0314 \\
\hline 4 & 1 & 0 & 187 & 181 & False & 0.0314 & -1 & -0.0314 \\
\hline 5 & 0 & 1 & 187 & 181 & False & 0.0314 & 1 & 0.0314 \\
\hline 6 & 1 & 0 & 187 & 181 & False & 0.0314 & -1 & -0.0314 \\
\hline 7 & 1 & 1 & 187 & 181 & False & 0 & 1 & 0 \\
\hline 8 & 1 & 0 & 187 & 181 & False & 0.0314 & -1 & -0.0314 \\
\hline 9 & 1 & 0 & 187 & 181 & False & 0.0314 & -1 & -0.0314 \\
\hline 10 & 0 & 1 & 187 & 181 & False & 0.0314 & 1 & 0.0314 \\
\hline 11 & 0 & 0 & 187 & 181 & False & 0 & 1 & 0 \\
\hline 12 & 0 & 1 & 187 & 181 & False & 0.0314 & 1 & 0.0314 \\
\hline 13 & 0 & 0 & 187 & 181 & False & 0 & 1 & 0 \\
\hline 14 & 1 & 1 & 187 & 181 & False & 0 & 1 & 0 \\
\hline 15 & 0 & 1 & 187 & 181 & False & 0.0314 & 1 & 0.0314 \\
\hline
\end{tabular}

Figure 21. $\theta_{1 k l}^{0}$ of quantum bits of $q t 5 v_{1}^{0}$ in $Q_{T 5 V}(0)$.

\begin{tabular}{|c|c|c|c|c|c|c|c|c|c|c|c|c|c|c|c|}
\hline & & & & & & & & & & & & & & & \\
\hline & & & & & & & & & & & & & & & \\
\hline \multirow{2}{*}{$5 v_{1}^{1}$} & & & & & & & & & & & & & & & \\
\hline & & & & & & & & & & & & & & & \\
\hline \multirow{2}{*}{$5 v_{2}{ }^{1}$} & & & & & & & & & & & & & & & \\
\hline & 2 & & & ( & & & & & & & & & & & \\
\hline \multirow{2}{*}{$v_{3}{ }^{1}$} & & & & & & & & & & & & & & & \\
\hline & ( & & & & & & & & & & & & & & \\
\hline \multirow{2}{*}{$v_{4}^{1}$} & & & & ( & & & & & & & & & & & \\
\hline & 071 & & & 9 & & & 2 & & & & & & & & \\
\hline \multirow{2}{*}{$v_{5}{ }^{1}$} & & & & & & & & & & & & & & & \\
\hline & & & & & & & & & & & & & & & \\
\hline \multirow{2}{*}{$5 v_{6}^{1}$} & 720 & & & 1 & & 1 & 6 & & & & & & & & \\
\hline & 071 & & & 7071 & & 71 & 2 & & & & & & & & 29 \\
\hline \multirow{2}{*}{$v_{7}^{1}$} & 071 & & & 7071 & & 7071 & 7071 & & 71 & & & & & & 48 \\
\hline & 071 & & & & & & & & & & & & & & $10 / 1$ \\
\hline \multirow{2}{*}{$5 v_{8}^{1}$} & & & & 0 & & 0 & & & & & & & & & \\
\hline & 282 & & & & & & & & & & & & & & \\
\hline & 7057 & & & 0.7057 & & 39 & 0 & & 57 & & & & & & 726 \\
\hline & 289 & & & 0 & & 0.7071 & 1 & & & & & & & & \\
\hline \multirow[b]{2}{*}{$v_{10}$} & 064 & & 0.6846 & 0.6846 & & 0.7290 & 0.6846 & 0.7065 & 7065 & 71 & & & 346 & 290 & 84 \\
\hline & 7289 & & 0.7282 & 0.7071 & 0.1289 & 0.6839 & 0.7282 & 0.6846 & 0.6846 & 0.7071 & 0.7071 & 0.7282 & 0.7282 & 0.083 & 0.728 \\
\hline
\end{tabular}

Figure 22. $Q_{T 5 V}(1)$.

Step-5 to Step-9 are repeated for a pre-specified number of generations $G$, whereafter the Top-5 views are produced as output.

Next, experiment-based comparison of QIEVSA to the evolutionary view selection algorithms DEVSA, MVSA, GVSA and HRUA is discussed.

\section{Experimental results}

Algorithms QIEVSA, DEVSA, GVSA, MVSA and HRUA were implemented using $J D K 1.7$ in a Windows-7 environment on an Intel-based $2.13 \mathrm{GHz} \mathrm{PC}$ having $4 \mathrm{~GB}$ RAM. First, the appropriate value of number of generations 


\begin{tabular}{|c|c|c|c|c|c|}
\hline$B_{T 5 \nu}(1)$ & $b v_{i 1}^{1}$ & $b v_{i 2}^{1}$ & $b v_{i 3}^{1}$ & $b v_{i 4}^{1}$ & $b v_{i 5}^{1}$ \\
\hline$b t 5 v_{I}^{I}$ & 001 & 011 & 110 & 010 & 100 \\
\hline$b t 5 v_{2}^{I}$ & 111 & 010 & 100 & 101 & 011 \\
\hline$b t 5 v_{3}^{I}$ & 101 & 110 & 010 & 111 & 001 \\
\hline$b t 5 v_{4}^{I}$ & 001 & 111 & 100 & 110 & 101 \\
\hline$b t 5 v_{5}^{I}$ & 010 & 101 & 001 & 111 & 110 \\
\hline$b t 5 v_{6}^{I}$ & 001 & 100 & 111 & 101 & 110 \\
\hline$b t 5 v_{7}^{I}$ & 111 & 001 & 011 & 101 & 010 \\
\hline$b t 5 v_{8}^{I}$ & 001 & 110 & 100 & 101 & 000 \\
\hline$b t 5 v_{9}^{I}$ & 001 & 011 & 010 & 101 & 111 \\
\hline$b t 5 v_{10}{ }^{I}$ & 101 & 110 & 100 & 001 & 111 \\
\hline
\end{tabular}

Figure 23. $B_{T 5 V}(1)$.

\begin{tabular}{|c|c|c|c|c|c|c|}
\hline$D_{\text {TK } v}(1)$ & $d v_{i 1}^{l}$ & $d v_{i 2}^{l}$ & $d v_{i 3}^{l}$ & $d v_{i 4}^{l}$ & $d v_{i 5}^{l}$ & $T V E C$ \\
\hline$d t 5 v_{1}{ }^{l}$ & 1 & 3 & 6 & 2 & 4 & 174 \\
\hline$d t 5 v_{2}{ }^{l}$ & 5 & 6 & 2 & 7 & 1 & 183 \\
\hline$d t 5 v_{3}{ }^{l}$ & 7 & 2 & 4 & 5 & 3 & 181 \\
\hline$d t 5 v_{4}{ }^{l}$ & 1 & 7 & 4 & 6 & 5 & 183 \\
\hline$d t 5 v_{s}{ }^{l}$ & 2 & 5 & 1 & 7 & 6 & 183 \\
\hline$d t 5 v_{6}{ }^{l}$ & 1 & 4 & 7 & 5 & 6 & 183 \\
\hline$d t 5 v_{7}{ }^{I}$ & 7 & 1 & 3 & 5 & 2 & 183 \\
\hline$d t 5 v_{s}{ }^{l}$ & 1 & 6 & 4 & 5 & 7 & 183 \\
\hline$d t 5 v_{9}{ }^{l}$ & 1 & 3 & 2 & 5 & 7 & 183 \\
\hline$d t 5 v_{10}{ }^{l}$ & 5 & 6 & 4 & 1 & 7 & 183 \\
\hline
\end{tabular}

Figure 24. $D_{T 5 V}(1)$.

\begin{tabular}{|c|c|c|c|c|c|c|}
\hline Top-5 views & View-1 & View-2 & View-3 & View-4 & View-5 & TVEC \\
\hline$B_{T S V}$ & 001 & 011 & 110 & 010 & 100 & 174 \\
\hline$D_{T S V}$ & 1 & 3 & 6 & 2 & 4 & 174 \\
\hline
\end{tabular}

Figure 25. $B_{T 5 V}$ and $D_{T 5 V}$.

\begin{tabular}{|c|c|c|c|c|c|}
\hline$D_{\text {TKV }}(1)$ & $d v_{i 1}^{1}$ & $d v_{i 2}^{1}$ & $d v_{i 3}^{1}$ & $d v_{i 4}^{1}$ & $d v_{i 5}^{1}$ \\
\hline$d t 5 v_{1}^{1}$ & 1 & 3 & 6 & 2 & 4 \\
\hline$d t 5 v_{1}^{1}$ & 1 & 3 & 6 & 2 & 4 \\
\hline$d t 5 v_{3}{ }^{1}$ & 7 & 2 & 4 & 5 & 3 \\
\hline$d t 5 v_{3}{ }^{1}$ & 7 & 2 & 4 & 5 & 3 \\
\hline$d t 5 v_{5}{ }^{1}$ & 2 & 5 & 1 & 7 & 6 \\
\hline$d t 5 v_{5}{ }^{1}$ & 2 & 5 & 1 & 7 & 6 \\
\hline$d t 5 v_{7}{ }^{1}$ & 7 & 1 & 3 & 5 & 2 \\
\hline$d t 5 v_{7}{ }^{1}$ & 7 & 1 & 3 & 5 & 2 \\
\hline$d t 5 v_{10}{ }^{1}$ & 5 & 6 & 4 & 1 & 7 \\
\hline$d t 5 v_{10}{ }^{1}$ & 5 & 6 & 4 & 1 & 7 \\
\hline
\end{tabular}

Figure 26. $D_{T 5 V}(1)$ after local migration.

after which local migration $G_{L M}$ and global migration $G_{G M}$ are to be performed, for which QIEVSA is able to select views having lower TVEC, is determined. The mean value of TVEC of Top-10 views over four simulation runs after each generation up to 500 generations for various combinations of $G_{L M}$ and $G_{G M}$ was used for plotting graphs [98]. These graphs showing the TVEC of the Top-10 views for $\left(G_{L M}, G_{G M}\right)=\{(1,50),(1,100),(2,50),(2,100),(4,50)$,

\section{QIEVSA \\ (5 Dimensions, Top-10 Views)}

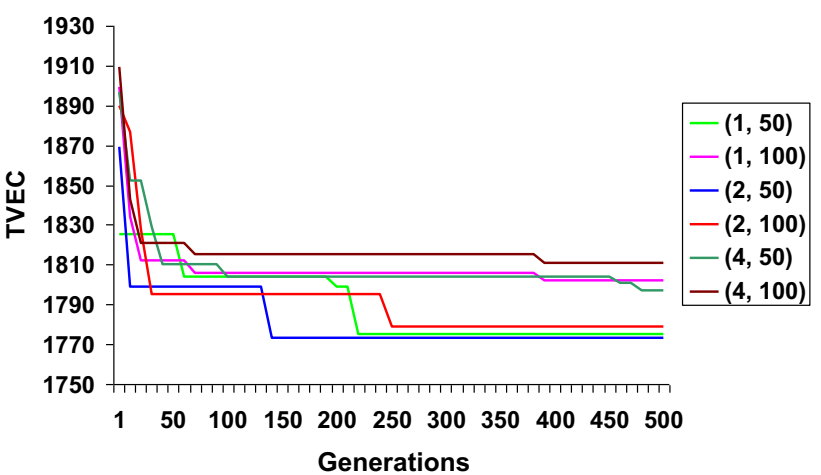

Figure 27. QIEVSA - TVEC Vs. Generations - Top-10 Views - 5 Dimensions.

QIEVSA

(6 Dimensions, Top-10 Views)

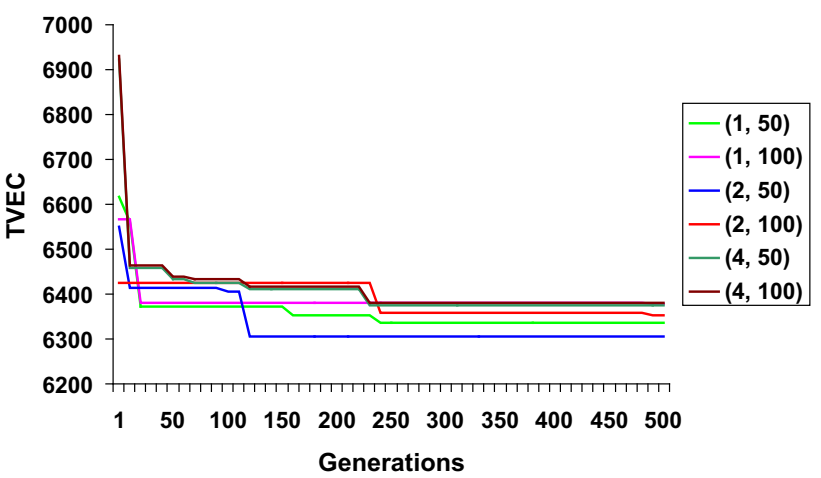

Figure 28. QIEVSA-TVEC vs. generations - Top-10 views -6 dimensions.

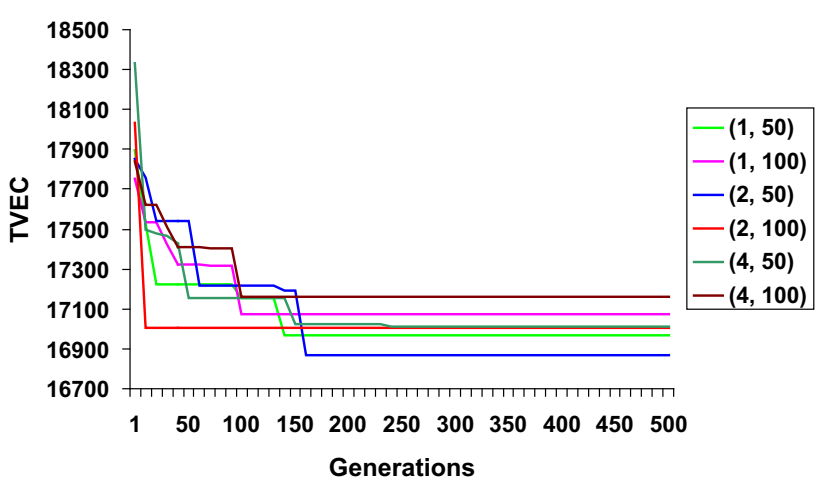

Figure 29. QIEVSA-TVEC vs. generations - Top-10 views - 7 dimensions. 
QIEVSA

(8 Dimensions, Top-10 Views)

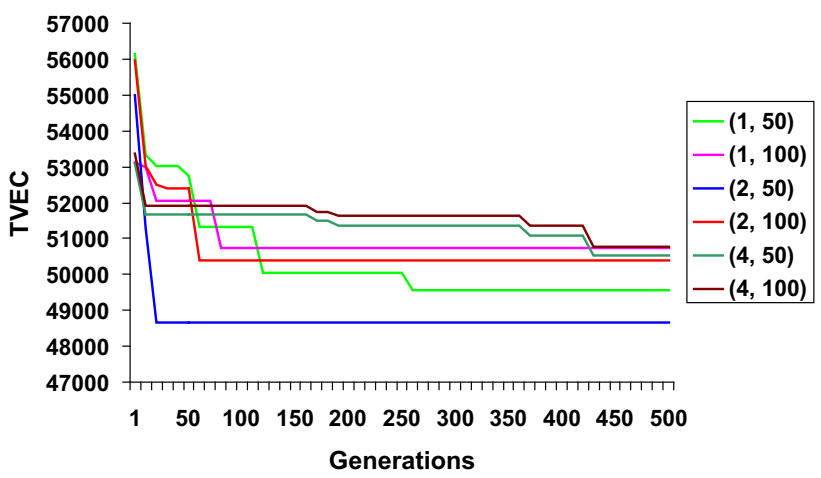

Figure 30. QIEVSA-TVEC vs. generations - Top-10 views - 8 dimensions.

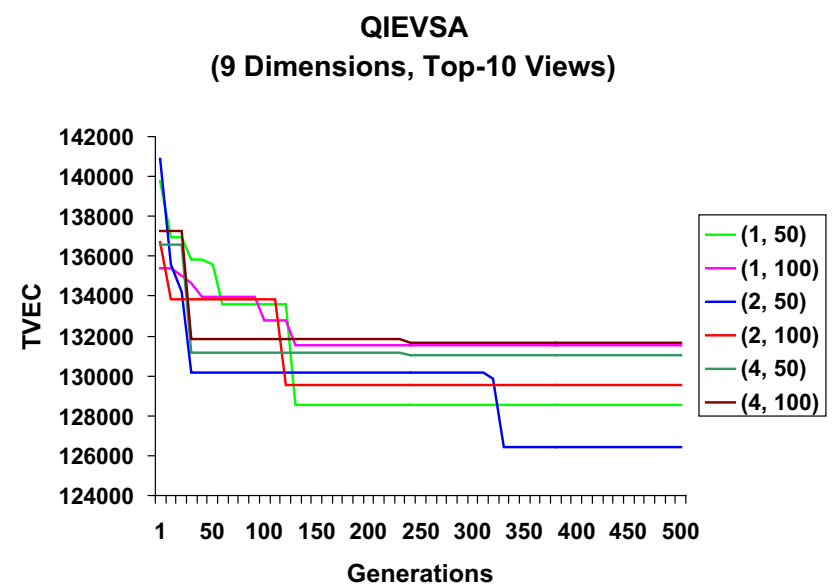

Figure 31. QIEVSA-TVEC vs. generations - Top-10 views - 9 dimensions.

QIEVSA

(10 Dimensions, Top-10 Views)

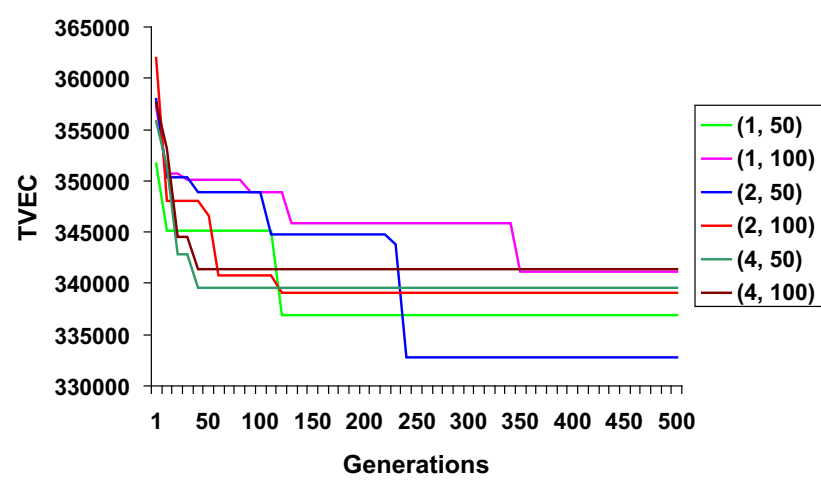

Figure 32. QIEVSA-TVEC vs. generations - Top-10 views -10 dimensions.

\begin{tabular}{|c|c|c|c|c|}
\hline \multicolumn{5}{|c|}{ Dimension $=5$} \\
\hline$\left(G_{L M}, G_{G M}\right)$ & $\operatorname{minTVEC}$ & maxTVEC & meanTVEC & stdTVEC \\
\hline$(1,50)$ & 1755 & 1802 & 1777.25 & 20.9687 \\
\hline$(1,100)$ & 1780 & 1824 & 1801.50 & 17.0953 \\
\hline$(2,50)$ & 1752 & 1794 & 1773.25 & 15.8646 \\
\hline$(2,100)$ & 1759 & 1801 & 1779.25 & 18.8994 \\
\hline$(4,50)$ & 1768 & 1823 & 1796.75 & 24.0767 \\
\hline$(4,100)$ & 1784 & 1835 & 1810.50 & 20.7665 \\
\hline \multicolumn{5}{|c|}{ Dimension $=6$} \\
\hline$\left(G_{L M}, G_{G M}\right)$ & $\operatorname{minTVEC}$ & $\operatorname{maxTVEC}$ & meanTVEC & stdTVEC \\
\hline$(1,50)$ & 6303 & 6369 & 6336.25 & 30.3757 \\
\hline$(1,100)$ & 6345 & 6408 & 6376.50 & 27.3724 \\
\hline$(2,50)$ & 6281 & 6334 & 6306.25 & 21.2294 \\
\hline$(2,100)$ & 6312 & 6390 & 6352.25 & 33.7667 \\
\hline$(4,50)$ & 6341 & 6404 & 6374.50 & 26.6505 \\
\hline$(4,100)$ & 6349 & 6424 & 6381.25 & 30.1859 \\
\hline \multicolumn{5}{|c|}{ Dimension $=7$} \\
\hline$\left(G_{L M}, G_{G M}\right)$ & $\operatorname{minTVEC}$ & $\operatorname{maxTVEC}$ & meanTVEC & stdTVEC \\
\hline$(1,50)$ & 16875 & 17087 & 16965.25 & 83.6313 \\
\hline$(1,100)$ & 16979 & 17165 & 17071.50 & 85.8298 \\
\hline$(2,50)$ & 16813 & 16956 & 16868.25 & 57.1856 \\
\hline$(2,100)$ & 16924 & 17089 & 17004.25 & 75.1844 \\
\hline$(4,50)$ & 16937 & 17081 & 17013.75 & 60.4126 \\
\hline$(4,100)$ & 17059 & 17227 & 17157.50 & 69.5899 \\
\hline \multicolumn{5}{|c|}{ Dimension $=8$} \\
\hline$\left(G_{L M}, G_{G M}\right)$ & $\operatorname{minTVEC}$ & $\operatorname{maxTVEC}$ & meanTVEC & stdTVEC \\
\hline$(1,50)$ & 49415 & 49665 & 49551.50 & 106.3332 \\
\hline$(1,100)$ & 50601 & 50899 & 50746.75 & 127.5233 \\
\hline$(2,50)$ & 48561 & 48763 & 48669.75 & 85.0922 \\
\hline$(2,100)$ & 50285 & 50517 & 50398.75 & 108.5504 \\
\hline$(4,50)$ & 50455 & 50644 & 50531.25 & 106.2835 \\
\hline$(4,100)$ & 50651 & 50915 & 50785.25 & 112.3975 \\
\hline \multicolumn{5}{|c|}{ Dimension $=9$} \\
\hline$\left(G_{L M}, G_{G M}\right)$ & $\min T V E C$ & $\operatorname{maxTVEC}$ & meanTVEC & stdTVEC \\
\hline$(1,50)$ & 128311 & 128787 & 128559.50 & 201.8831 \\
\hline$(1,100)$ & 131230 & 131787 & 131539.50 & 228.3533 \\
\hline$(2,50)$ & 126241 & 126613 & 126417.25 & 171.2547 \\
\hline$(2,100)$ & 129311 & 129749 & 129512.75 & 211.1378 \\
\hline$(4,50)$ & 130811 & 131273 & 131024.25 & 204.6306 \\
\hline$(4,100)$ & 131379 & 131941 & 131682.25 & 225.5076 \\
\hline \multicolumn{5}{|c|}{ Dimension $=10$} \\
\hline$\left(G_{L M}, G_{G M}\right)$ & $\operatorname{minTVEC}$ & $\operatorname{maxTVEC}$ & meanTVEC & stdTVEC \\
\hline$(1,50)$ & 336255 & 337557 & 336860.75 & 574.3424 \\
\hline$(1,100)$ & 340445 & 341787 & 341094.50 & 593.7935 \\
\hline$(2,50)$ & 332181 & 333141 & 332745.75 & 422.6851 \\
\hline$(2,100)$ & 338715 & 339654 & 339088.75 & 524.4666 \\
\hline$(4,50)$ & 339141 & 340165 & 339621.25 & 522.8061 \\
\hline$(4,100)$ & 340611 & 341981 & 341328.25 & 556.3926 \\
\hline
\end{tabular}

Figure 33. QIEVSA-minTVEC, maxTVEC, meanTVEC, stdTVEC - Top-10 views.

$(4,100)\}$ for dimensions $5,6,7,8,9$ and 10 are shown in figures 27, 28, 29, 30, 31 and 32, respectively. From each of these graphs, it can be inferred that the Top-10 views selected by QIEVSA have a lower TVEC for $G_{L M}=2$ and $G_{G M}=50$. Further, minTVEC (minimum value of TVEC), maxTVEC (maximum value of TVEC), meanTVEC (mean value of TVEC) and the stdTVEC (standard deviation of 
QIEVSA Vs. DEVSA Vs. MVSA Vs. GVSA Vs. HRUA

(5 Dimensions)

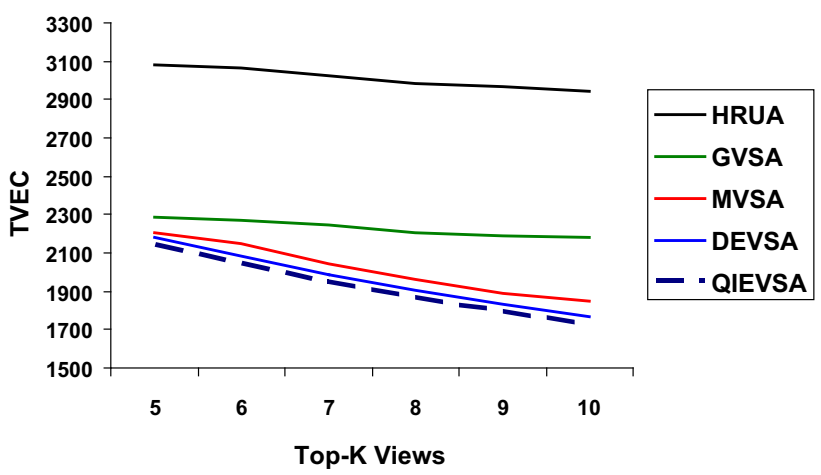

Figure 34. $Q I E V S A$ vs. DEVSA vs. $M V S A$ vs. GVSA vs. HRUA$T V E C$ vs. Top $-K$ views -5 dimensions.

QIEVSA Vs. DEVSA Vs. MVSA Vs. GVSA Vs. HRUA (6 Dimensions)

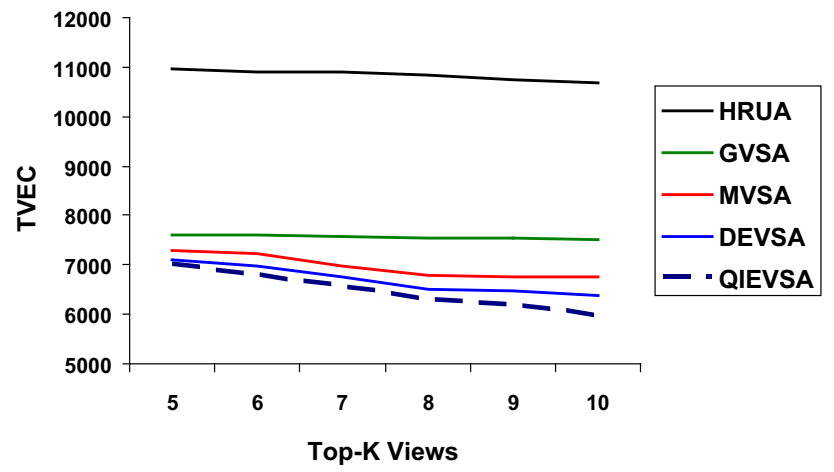

Figure 35. $Q I E V S A$ vs. DEVSA vs. $M V S A$ vs. $G V S A$ vs. $H R U A-$ $T V E C$ vs. Top $-K$ views -6 dimensions.

QIEVSA Vs. DEVSA Vs. MVSA Vs. GVSA Vs. HRUA (7 Dimensions)

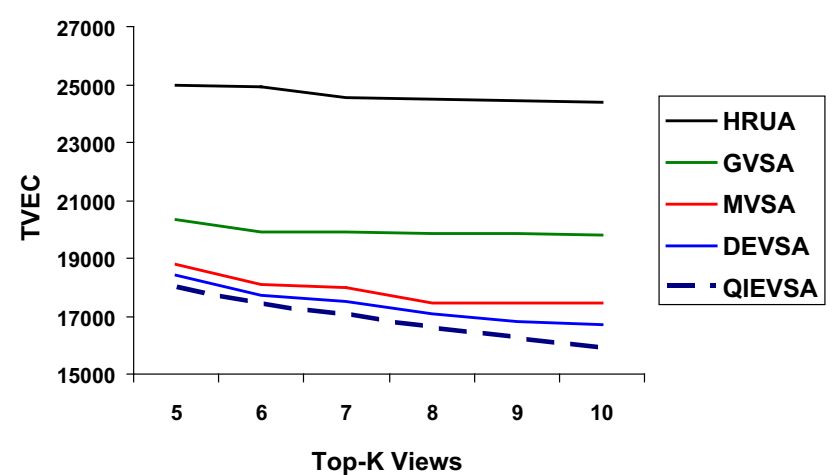

Figure 36. $Q I E V S A$ vs. DEVSA vs. $M V S A$ vs. GVSA vs. HRUA$T V E C$ vs. Top- $K$ views -7 dimensions.
QIEVSA Vs. DEVSA Vs. MVSA Vs. GVSA Vs. HRUA

(8 Dimensions)

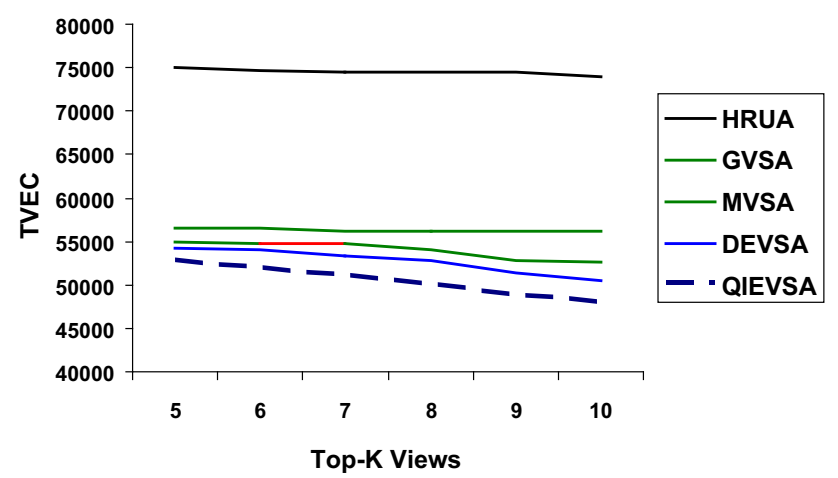

Figure 37. $Q I E V S A$ vs. DEVSA vs. $M V S A$ vs. GVSA vs. HRUA$T V E C$ vs. Top $-K$ views -8 dimensions.

QIEVSA Vs. DEVSA Vs. MVSA Vs. GVSA Vs. HRUA
(9 Dimensions)

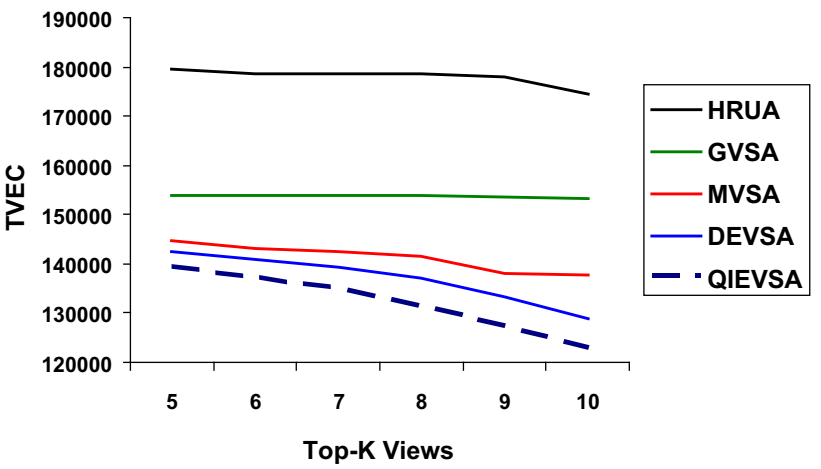

Figure 38. $Q I E V S A$ vs. DEVSA vs. $M V S A$ vs. GVSA vs. HRUA$T V E C$ vs. Top $K$ views -9 dimensions.

QIEVSA Vs. DEVSA Vs. MVSA Vs. GVSA Vs. HRUA (10 Dimensions)

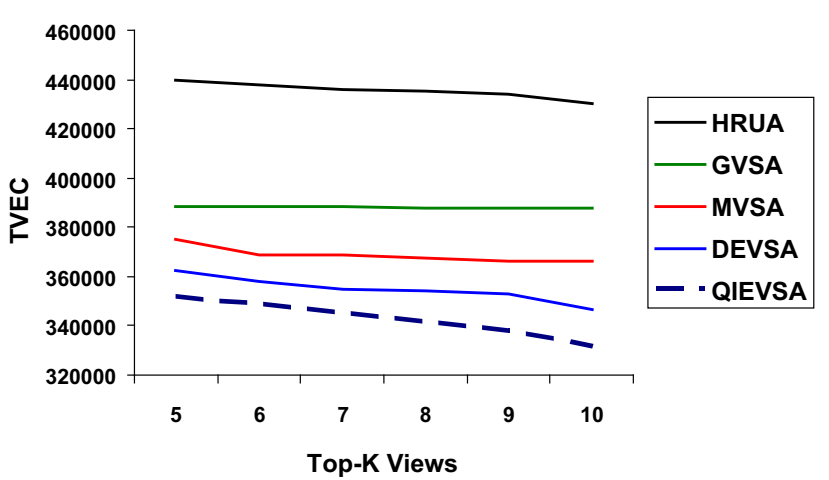

Figure 39. $Q I E V S A$ vs. DEVSA vs. $M V S A$ vs. GVSA vs. HRUA$T V E C$ vs. Top- $K$ views -10 dimensions. 


\begin{tabular}{|c|c|c|c|c|c|}
\hline \multicolumn{6}{|c|}{ Dimension $=5$} \\
\hline Views & $H R U A$ & GVSA & $M V S A$ & $D E V S A$ & QIEVSA \\
\hline Top-5 & 3082 & 2287 & 2203 & 2182 & 2140 \\
\hline Top-6 & 3064 & 2270 & 2147 & 2081 & 2045 \\
\hline Top-7 & 3024 & 2249 & 2044 & 1985 & 1948 \\
\hline Top-8 & 2984 & 2208 & 1960 & 1905 & 1864 \\
\hline Top-9 & 2964 & 2190 & 1890 & 1831 & 1795 \\
\hline Top-10 & 2944 & 2182 & 1850 & 1766 & 1726 \\
\hline \multicolumn{6}{|c|}{ Dimension $=6$} \\
\hline Views & HRUA & GVSA & $M V S A$ & DEVSA & QIEVSA \\
\hline Top-5 & 10972 & 7603 & 7293 & 7098 & 6998 \\
\hline Top-6 & 10908 & 7597 & 7244 & 6991 & 6791 \\
\hline Top-7 & 10905 & 7574 & 6985 & 6768 & 6568 \\
\hline Top-8 & 10832 & 7540 & 6775 & 6519 & 6278 \\
\hline Top-9 & 10737 & 7543 & 6765 & 6485 & 6206 \\
\hline Top-10 & 10688 & 7526 & 6751 & 6394 & 5984 \\
\hline \multicolumn{6}{|c|}{ Dimension $=7$} \\
\hline Views & HRUA & GVSA & MVSA & DEVSA & QIEVSA \\
\hline Top-5 & 24964 & 20344 & 18787 & 18406 & 17996 \\
\hline Top-6 & 24904 & 19907 & 18104 & 17694 & 17389 \\
\hline Top-7 & 24552 & 19898 & 17978 & 17508 & 17046 \\
\hline Top-8 & 24476 & 19879 & 17475 & 17105 & 16587 \\
\hline Top-9 & 24462 & 19868 & 17464 & 16835 & 16229 \\
\hline Top-10 & 24384 & 19786 & 17453 & 16710 & 15898 \\
\hline \multicolumn{6}{|c|}{ Dimension $=8$} \\
\hline Views & HRUA & GVSA & $M V S A$ & DEVSA & QIEVSA \\
\hline Top-5 & 74993 & 56620 & 55016 & 54206 & 52798 \\
\hline Top-6 & 74742 & 56468 & 54814 & 53981 & 51871 \\
\hline Top-7 & 74525 & 56240 & 54682 & 53268 & 50969 \\
\hline Top-8 & 74491 & 56228 & 53980 & 52851 & 49882 \\
\hline Top-9 & 74489 & 56168 & 52837 & 51405 & 48887 \\
\hline Top-10 & 73984 & 56159 & 52589 & 50573 & 47926 \\
\hline \multicolumn{6}{|c|}{ Dimension $=9$} \\
\hline Views & HRUA & GVSA & MVSA & DEVSA & QIEVSA \\
\hline Top-5 & 179577 & 153975 & 144570 & 142507 & 139432 \\
\hline Top-6 & 178662 & 153765 & 143259 & 140767 & 137143 \\
\hline Top-7 & 178652 & 153764 & 142481 & 139413 & 134856 \\
\hline Top-8 & 178478 & 153738 & 141554 & 137030 & 131455 \\
\hline Top-9 & 177950 & 153682 & 138212 & 133219 & 127219 \\
\hline Top-10 & 174592 & 153369 & 137612 & 128985 & 122985 \\
\hline \multicolumn{6}{|c|}{ Dimension $=10$} \\
\hline Views & HRUA & GVSA & $M V S A$ & DEVSA & QIEVSA \\
\hline Top-5 & 439504 & 388733 & 375200 & 362483 & 351456 \\
\hline Top-6 & 438066 & 388359 & 368866 & 358228 & 348696 \\
\hline Top-7 & 436078 & 388250 & 368736 & 354751 & 345574 \\
\hline Top-8 & 435098 & 387970 & 367671 & 354120 & 341691 \\
\hline Top-9 & 433758 & 387798 & 366483 & 353032 & 337457 \\
\hline Top-10 & 430336 & 387737 & 365948 & 346765 & 331289 \\
\hline
\end{tabular}

Figure 40. $Q I E V S A$ vs. DEVSA vs. $M V S A$ vs. GVSA vs. HRUA (TVEC of Top- $K$ views for 5-10 dimensions).
TVEC) over four simulation runs for selecting Top-10 views after 500 generations are computed for dimensions 5-10.They are given in a table in figure 33. From the table also, it can be observed that QIEVSA performs best for $G_{L M}=2$ and $G_{G M}=50$. These values of $G_{L M}$ and $G_{G M}$ for QIEVSA were used for further comparisons to DEVSA, GVSA, MVSA and HRUA.

Next, TVECs of the Top- $K(K=5,6,7,8,9,10)$ views selected using HRUA, GVSA, MVSA, DEVSA and QIEVSA for dimensions $5,6,7,8,9$ and 10 are plotted and are shown in figures $34,35,36,37,38$ and 39 , respectively. The comparisons were based on the values GVSA (crossover probability $P_{c}=0.6$, mutation probability $P_{m}=0.05$ ) observed in [64], MVSA (crossover probability $P_{c}=0.8$, mutation probability $P_{m}=0.05$ ) observed in [65], DEVSA (crossover rate $C R=0.6$, scaling factor $F=0.1$ ) observed in [66] and QIEVSA $\left(G_{L M}=2, G_{G M}=50\right)$ observed from figure 33. The mean of the TVEC values, for each of the five algorithms over four simulation runs, was taken for plotting graphs. It can be inferred from each of these graphs that the Top-K views selected using QIEVSA, in comparison with those selected using DEVSA, MVSA, GVSA and HRUA, have a lower TVEC. Further, it can be observed from figure 40 that the difference in the TVEC value increases with increase in the dimensions and the value of $K$. Furthermore, the performance of DEVSA is the next best followed by MVSA and GVSA. Views selected using HRUA, in comparison with others, have a higher TVEC.

\section{Conclusions}

In this paper, a QIEA has been suitably adapted and discretized to address the view selection problem in a multidimensional lattice framework. Accordingly, view selection algorithm QIEVSA that selects the Top- $K$ views from a multidimensional lattice has been proposed. The Q-bits, Q-gates and the observation process in QIEA have been suitably adapted and discretized in QIEVSA to generate a population of Top- $K$ views for the subsequent generation. QIEVSA, at first, randomly selects a population of Q-bit Top- $K$ views. Binary sets of Top- $K$ views are generated by observing the quantum state of the Top- $K$ views in the population. TVEC of these views is then computed, whereafter the best set of Top-K views are updated. Thereafter, the Q-bit Top- $K$ views are updated by applying the rotation Q-gate operator. QIEVSA terminates after running for a pre-specified number of generations, whereupon the Top-K views having minimum TVEC are produced as output. Further, experimental comparison of QIEVSA, with other evolutionary view selection algorithms based on multidimensional lattice framework like DEVSA, MVSA, GVSA and HRUA, shows that QIEVSA is able to select Top- $K$ views at a comparatively lesser TVEC for the observed values of $G_{L M}$ and $G_{G M}$. This performance 
improves for higher dimensions. Further, QIEVSA is able select views for higher dimensional data sets, as, in QIEVSA, population of the Top-K views is randomly generated and their TVEC is computed from the lattice. This is unlike the case in $H R U A$, where $H R U A$ needs to compute the $T o p-K$ views from an exponentially large search space of possible views and for higher dimensional data sets, it becomes almost infeasible to select views for materialization using $H R U A$. Thus, it can be reasonably inferred that QIEVSA is able to select reasonably good quality views, for higher dimensional data sets, that are capable of reducing the response time of analytical queries, which thereby would lead to efficient decision making. As future work, QIEVSA would be compared to existing swarm-based view selection algorithms [99-107].

\section{References}

[1] Inmon W H 2003 Building the data warehouse, 3rd ed. India: Wiley Dreamtech India Pvt. Ltd

[2] Rainardi V 2008 Building a data warehouse: with examples in SQL server. New York: Apress

[3] Kimball R and Ross M 2002 The data warehouse toolkit: the complete guide to dimensional modelling. Hoboken: Wiley

[4] Widom J 1995 Research problems in data warehousing. In: Proceedings on the 4th international conference on information and knowledge management, Baltimore, Maryland, pp. $25-30$

[5] Ponniah P 2004 Data warehousing fundamentals: a comprehensive guide for IT professionals. Hoboken: Wiley

[6] Cabibbo L and Torlone R 1998 A logical approach to multidimensional databases. In: Advances in database technology-EDBT'98, Lecture notes in computer science, vol. 1377, pp. 183-197

[7] Choong Y W, Laurent A and Laurent D 2007 Pixelizing data cubes: a block-based approach. In: Pixelization paradigm, Lecture notes in computer science, vol. 4370, pp. 63-76

[8] Codd E F, Codd S B and Salley C T 1993 Providing OLAP (on line analytical processing) to user-analysts: an IT mandate. Dorset: E. F. Codd and Associates

[9] Thomsen E 2002 OLAP solutions: building multidimensional information systems. Hoboken: Wiley

[10] Golfarellia M, Maniezzo V and Rizzi S 2004 Materialization of fragmented views in multidimensional databases. Data Knowl. Eng. 49: 325-351

[11] Gray J, Bosworth A, Lyaman A and Pirahesh H 1996 Data cube: a relational aggregation operator generalizing GROUP-BY, CROSS-TAB, and SUB-TOTALS. In: Proceedings of the 12th IEEE international conference on data engineering, pp. 152-159

[12] Gupta H 1997 Selection of views to materialize in a data warehouse. In: Proceedings of the 6th ICDT, pp. 98-112

[13] Harinarayan V, Rajaraman A and Ullman J D 1996 Implementing data cubes efficiently. In: Proceedings of ACM SIGMOD, Montreal, Canada, pp. 205-216
[14] Niemi T, Nummenmaa J and Thanisch P 2001 Constructing OLAP cubes based on queries. In: Proceedings of the 4th ACM international workshop on data warehousing and OLAP, pp. 9-15

[15] Roussopoulos N 1997 Materialized views and data warehouse. In: Proceedings of the 4th KRDB workshop, Athens, Greece, August

[16] Chirkova R and Yang J 2011 Materialized views. Found. Trends Databases 4(4): 295-405

[17] Mami I and Bellahsene Z 2012 A survey of view selection methods. ACM SIGMOD Record 41(1): 20-29

[18] Yang J, Karlapalem K and Li Q 1997 Algorithms for materialized view design in data warehousing environment. Very Large databases (VLDB) J 136-145

[19] Yousri N A R, Ahmed K M and El-Makky N M 2005 Algorithms for selecting materialized views in a data warehouse. In: Proceedings of the ACS/IEEE 2005 international conference on computer systems and applications, AICCSA'05, IEEE Computer Society, pp. 27-1

[20] Chirkova R, Halevy A Y and Suciu D 2001 A formal perspective on the view selection problem. In: Proceedings of the 27 VLDB conference, Italy, pp. 59-68

[21] Chaves L W F, Buchmann E, Hueske F and Bohm K 2009 Towards materialized view selection for distributed databases. In: Proceedings of the 12th international conference on extending database technology: advances in database technology, pp. 1088-1099

[22] Goasdoue F, Karanasos K, Leblay J and Manolescu I 2011 View selection in semantic web databases. In: Proceedings of the VLDB endowment, vol. 5(2), pp. 97-108

[23] Katsifodimos A, Manolescu I and Vassalos V 2012 Materialized view selection for XQuery workloads. In: Proceedings of the international conference on management of data (SIGMOD'12), pp. 565-576

[24] Teschke M and Ulbrich A 1997 Using materialized views to speed up data warehousing. Technical Report, IMMD 6, Universität Erlangen-Nümberg

[25] Agrawal S, Chaudhuri S and Narasayya V R 2000 Automated selection of materialized views and indexes in SQL databases. In: Proceedings of the 26th international conference on very large databases, Cairo, Egypt, pp. 496-505

[26] Aouiche K and Darmont J 2009 Data mining-based materialized view and index selection in data warehouse. $J$. Intell. Inf. Syst. 33(1): 65-93

[27] Aouiche K, Jouve P E and Darmont J 2006 Clusteringbased materialized view selection in data warehouses. In: Proceedings of the 10th East-European conference on advances in databases and information systems (ADBISO6), Thessaloniki, Greece, LNCS, vol. 4152, pp. 81-95

[28] Baralis E, Paraboschi S and Teniente E 1997 Materialized view selection in a multidimensional database. In: Proceedings of the 23rd VLDB conference, Greece, pp. 156-165

[29] Lehner W, Ruf T and Teschke M 1996 Improving query response time in scientific databases using data aggregation. In: Proceedings of the 7th international conference and workshop on database and expert systems applications, DEXA 96, Zurich, pp. 201-206

[30] Theodoratos D and Sellis T 1997 Data warehouse configuration. In: Proceedings of $V L D B$, Athens, Greece, pp. 126-135 
[31] Vijay Kumar T V and Devi K 2013 An architectural framework for constructing materialized views in a data warehouse. Int. J. Innov. Manag. Technol. 4(2): 192-197

[32] Vijay Kumar T V and Devi K 2012 Materialized view construction in data warehouse for decision making. Int $J$ Bus Inf Syst 11(4): 379-396

[33] Vijay Kumar T V, Goel A and Jain N 2010 Mining information for constructing materialized views. Int. J. Inf. Commun. Technol. 2(4): 386-405

[34] Gupta H and Mumick I S 2005 Selection of views to materialize in a data warehouse. IEEE Trans. Knowl. Data Eng. 17(1): 24-43

[35] Gupta H, Harinarayan V, Rajaraman V and Ullman J 1997 Index selection for OLAP. In: Proceedings of the 13th international conference on data engineering, ICDE 97, Birmingham, UK, pp. 208-219

[36] Haider M and Vijay Kumar T V 2011 Materialised view selection using size and query frequency. Int. J. Value Chain Manag. 5(2): 95-105

[37] Haider M and Vijay Kumar T V 2017 Query frequency based view selection. Int. J. Bus. Anal. 4(1): 36-55

[38] Serna-Encinas M T and Hoyo-Montano J A 2007 Algorithm for selection of materialized views: based on a costs model. In: Proceedings of the 8th international conference on current trends in computer science, pp. 18-24

[39] Shah B Ramachandran K and Raghavan V 2006 A hybrid approach for data warehouse view selection. Int. J. Data Wareh. Min. 2(2): 1-37

[40] Shukla A, Deshpande P M and Naughton J F 1998 Materialized view selection for multidimensional datasets. In: Proceedings of VLDB, pp. 488-500

[41] Valluri S, Vadapalli S and Karlapalem K 2002 View relevance driven materialized view selection in data warehousing environment. Aust. Comput. Sci. Commun. 24(2): 187-196

[42] Vijay Kumar T V and Ghoshal A 2009 A reduced lattice greedy algorithm for selecting materialized views. Commun. Comput. Inf. Sci. 31: 6-18

[43] Vijay Kumar T V, Haider M and Kumar S 2011 A view recommendation greedy algorithm for materialized views selection. Commun. Comput. Inf. Sci. 141: 61-70

[44] Vijay Kumar T V and Haider M 2010 A query answering greedy algorithm for selecting materialized views. In: Lecture notes in artificial intelligence (LNAI). Berlin: Springer, vol. 6422, pp. 153-162

[45] Vijay Kumar T V and Haider M 2011 Greedy views selection using size and query frequency. Commun. Comput. Inf. Sci. 125: 11-17

[46] Vijay Kumar T V and Haider M 2012 Materialized views selection for answering queries. In: Lecture notes in computer science (LNCS). Berlin: Springer, vol. 6411, pp. 44-51

[47] Vijay Kumar T V and Haider M 2015 Query answering based view selection. Int. J. Bus. Inf. Syst. 18(3): 338-353

[48] Vijay Kumar T V and Haider M 2011 Selection of views for materialization using size and query frequency. Commun. Comput. Inf. Sci. 147: 150-155

[49] Vijay Kumar T V, Haider M and Kumar S 2010 Proposing candidate views for materialization. Commun. Comput. Inf. Sci. 54: 89-98

[50] Vijay Kumar T V 2013 Answering query-based selection of materialised views. Int. J. Inf. Decision Sci. 5(1): 103-116
[51] De Jong K A 2006 Evolutionary computation: a unified approach. Cambridge: MIT Press

[52] Horng J T, Chang Y J, Liu B J and Kao C Y 1999 Materialized view selection using genetic algorithms in a data warehouse system. In: Proceedings of the world congress on evolutionary computation, IEEE CEC, Washington, DC, USA, vol. 3, pp. 2221-2227

[53] Lawrence M 2006 Multiobjective genetic algorithms for materialized view selection in OLAP data warehouses. In: Proceedings of the 8th annual conference on genetic and evolutionary computation, GECCO'06, July 8-12, Seattle, Washington, USA, pp. 699-706

[54] Lee M and Hammer J 2001 Speeding up materialized view selection in data warehouse using a random algorithm. Int. J. Coop. Inf. Syst. 10: 327-353

[55] Lin W and Kuo I C 2004 A genetic algorithm for OLAP data cubes. Int. J. Knowl. Inf. Syst. 6(1): 83-102

[56] Loureiro J and Belo O 2006 An evolutionary approach to the selection and allocation of distributed cubes. In: Proceedings of the 10th international database engineering and applications symposium, IDEAS'06, IEEE, pp. 243-248

[57] Talebian S H and Abdul Kareem S 2009 Using genetic algorithm to select materialized views subject to dual constraints. In: Proceedings of the international conference on signal processing systems, IEEE, pp. 633-638

[58] Wagner N and Agrawal V 2013 Using an evolutionary algorithm to solve the weighted view materialisation problem for data warehouses. Int. J. Intell. Inf. Database Syst. 7(2): 163-179

[59] Wang Z and Zhang D 2005 Optimal genetic view selection algorithm under space constraint. Int. J. Inf. Technol. 11(5): $44-51$

[60] Yu J X, Yao Y X, Choi C and Gou G 2003 Materialized view selection as constrained evolutionary optimization. IEEE Trans. Syst. Man Cybern. 33(4): 458-467

[61] Zhang C, Yao X and Yang J 2001 An evolutionary approach to materialized views selection in a data warehouse environment. IEEE Trans. Syst. Man Cybern. 31(3): 282-294

[62] Zhang C, Yao X and Yang J 1999 Evolving materialized views in a data warehouse. In: Proceedings of the IEEE congress on evolutionary computations, vol. 2, pp. 823-829

[63] Zhou L, He X and Li K 2012 An improved approach for materialized view selection based on genetic algorithm. $J$. Comput. 7(7): 1591-1598

[64] Vijay Kumar T V and Kumar S 2012 Materialized view selection using genetic algorithm. Commun. Comput. Inf. Sci. 306: 225-237

[65] Vijay Kumar T V and Kumar S 2013 Materialized view selection using memetic algorithm. In: Lecture notes in artificial intelligence (LNAI). Berlin: Springer, vol. 8284, pp. 316-327

[66] Vijay Kumar T V and Kumar S 2014 Materialized view selection using differential evolution. Int. J. Innov. Comput. Appl. 6(2):102-113

[67] Han K H and Kim J W 2002 Quantum-inspired evolutionary algorithm for a class of combinatorial optimization. IEEE Trans. Evolut. Comput. 6(6): 580-593

[68] Zhang G 2011 Quantum-inspired evolutionary algorithms: a survey and empirical study. J. Heuristics 17: 303-351 
[69] Manju A and Nigam M J 2014 Applications of quantum inspired computational intelligence: a survey. Artif. Intell. Rev. 42: 79-156

[70] Han K H, Park K H, Lee C H and Kim J H 2001 Parallel quantum inspired genetic algorithm for combinatorial optimization problem. In: Proceedings of the 2001 congress on evolutionary computation, Seoul, Korea, vol. 2, pp. 1422-1429

[71] Li B B and Wang L 2006 A hybrid quantum-inspired genetic algorithm for multi-objective scheduling. In: Proceedings of the 2006 international conference on intelligent computing, Kunming, China

[72] Narayan A and Moore M 1995 Quantum-inspired genetic algorithms. In: Proceedings of the 1996 IEEE international conference on evolutionary computation (ICEC96), pp. 61-66

[73] Zhou S and Sun Z 2005 A new approach belonging to EDAs: quantum-inspired genetic algorithm with only one chromosome. In: Lecture notes in computer science (LNCS). Berlin: Springer, vol. 3612, pp. 141-150

[74] Zhou R and Cao J 2014 Quantum novel genetic algorithm based on parallel subpopulation computing and its application. Artif Intell Rev 41(3): 359-371

[75] Hey T 1999 Quantum computing: an introduction. Comput. Control Eng. J. 10(3): 105-112

[76] Grigorenko I and Garcia M 2002 Calculation of the partition function using quantum genetic algorithms. Physica $A$ Stat. Mech. App. 313(3-4): 463-470

[77] Grigorenko I and Garcia M 2001 Ground-state wave functions of two-particle systems determined using quantum genetic algorithms. Physica A Stat. Mech. Appl. 291(1-4): 439-448

[78] Koza J R, Al-Sakran S H and Jones L W 2005 Crossdomain features of runs of genetic programming used to evolve designs for analog circuits, optical lens systems, controllers, antennas, mechanical systems, and quantum computing circuits. In: Proceedings of NASA/DoD EH, pp. 205-212

[79] Sahin M and Tomak M 2005 The self-consistent calculation of a spherical quantum dot: a quantum genetic algorithm study. Physica E Low Dimens. Syst. Nanostruct. 28(3): 247-256

[80] Spector L, Barnum H, Bernstein H and Swamy J N 1999 Finding a better-than-classical quantum AND/OR algorithm using genetic programming. In: Proceedings of the CEC, pp. 2239-2246

[81] Malossini A, Blanzieri E and Calarco T 2004 QGA: a quantum genetic algorithm. Technical Report No. DIT-04105, Informatica e Telecommunicazioni, University of Trento

[82] Rylander B, Soule T, Foster J and Alves-Foss J 2000 Quantum genetic algorithms. In: Proceedings of the GECCO, 373-377

[83] Sahin M, Atav U and Tomak M 2005 Quantum genetic algorithm method in self-consistent electronic structure calculations of a quantum dot with many electrons. Int. J. Mod. Phys. C16(9): 1379-1393

[84] Sofge D A 2006 Toward a framework for quantum evolutionary computation. In: Proceedings of the CIS conference, pp. 789-794

[85] Spector L, Barnum H and Bernstein H 1998 Genetic programming for quantum computers. In: Proceedings of the 3rd annual conference on genetic programming, pp. 365-373

[86] Udrescu M, Prodan L and Vladutiu M 2006 Implementing quantum genetic algorithms: a solution based on Grover's algorithm. In: Proceedings of $C F$, pp. 14-16

[87] Abs da Cruz A, Hall Barbosa C, Pacheco M and Vellasco M 2004 Quantum-inspired evolutionary algorithms and its application to numerical optimization problems. In: Proceedings of ICONIP 2004, LNCS 3316. Berlin: Springer, pp. 212-217

[88] Abs da Cruz A, Vellasco M and Pacheco M 2006 Quantuminspired evolutionary algorithm for numerical optimization. In: Proceedings of the CEC, pp. 2630-2637

[89] Han K and Kim J 2000 Genetic quantum algorithm and its application to combinatorial optimization problem. In: Proceedings of the CEC, vol. 2, pp. 1354-1360

[90] Han K and Kim J 2004 Quantum-inspired evolutionary algorithms with a new termination criterion, h-epsilon gate, and two-phase scheme. IEEE Trans. Evolut. Comput. 8(2): 156-169

[91] Liu H, Zhang G, Liu C and Fang C 2008 A novel memetic algorithm based on real-observation quantum inspired evolutionary algorithms. In: Proceedings of ISKE, pp. 486-490

[92] Sailesh Babu G S, Bhagwan Das D and Patvardhan C 2008 Real-parameter quantum evolutionary algorithm for economic load dispatch. IET Gener. Transm. Distrib. 2(1): 22-31

[93] Zhang G X and Rong H N 2007 Real-observation quantuminspired evolutionary algorithm for a class of numerical optimization problems. In: Lecture notes in computer science, vol. 4490, p. 996

[94] Kumar S 2015 Materialized view selection using randomized and evolutionary algorithms. Ph.D. Thesis, Jawaharlal Nehru University, New Delhi

[95] Vijay Kumar T V and Kumar S 2012 Materialized view selection using simulated annealing. In: Lecture notes in computer science (LNCS). Berlin: Springer, vol. 7678, pp. 168-179

[96] Vijay Kumar T V and Kumar S 2012 Materialized view selection using iterative improvement. Adv. Intell. Syst. Comput. 178: 205-214

[97] Vijay Kumar T V and Kumar S 2015 Materialized view selection using randomized algorithms. Int. J. Bus. Inf. Syst. 19(2): 224-240

[98] Derrac J, García S, Molina D and Herrera F 2011 A practical tutorial on the use of nonparametric statistical tests as a methodology for comparing evolutionary and swarm intelligence algorithms. Swarm Evolut. Comput. 1(1): 3-18

[99] Arun B and Vijay Kumar T V 2015 Materialized view selection using marriage in honey bees optimization. Int. J. Nat. Comput. Res. 5(3): 1-25

[100] Arun B and Vijay Kumar T V 2015 Materialized view selection using improvement based bee colony optimization. Int. J. Softw. Sci. Comput. Intell. 7(4): 35-61

[101] Vijay Kumar T V and Arun B 2016 Materialized view selection using BCO. Int. J. Bus. Inf. Syst. 22(3): 280-301

[102] Arun B and Vijay Kumar T V 2017 Materialized view selection using artificial bee colony optimization. Int. J. Intell. Inf. Technol. 13(1): 26-49 
[103] Arun B and Vijay Kumar T V 2017 Materialized view selection using bumble bee mating optimization. Int. J. Decision Support Syst. Technol. 9(3): 1-27

[104] Vijay Kumar T V and Arun B 2017 Materialized view selection using HBMO. Int. J. Syst. Assur. Eng. Manag. 8(1): 379-392

[105] Kumar A and Vijay Kumar T V 2017 Improved quality view selection for analytical query performance enhancement using particle swarm optimization. Int. J. Reliab.
Quality Saf. Eng. 24(6): https://doi.org/10.1142/ S0218539317400010

[106] Vijay Kumar T V, Kumar A and Arun B 2017 Cuckoo search based view selection. In: Emerging research in computing, information, communication and applications. Berlin: Springer, pp. 327-337

[107] Kumar A and Vijay Kumar T V 2018 Materialized view selection using set based particle swarm optimization. Int. J. Cogn. Inf. Nat. Intell. 12(3): 18-39 